\title{
Topology optimization for designing periodic microstructures based on finite strain viscoplasticity
}

\author{
Niklas Ivarsson ${ }^{1} \cdot$ Mathias Wallin ${ }^{1} \cdot$ Daniel A. Tortorelli ${ }^{2}$ \\ Received: 15 September 2019 / Revised: 20 February 2020 / Accepted: 21 February 2020 / Published online: 28 May 2020 \\ (C) The Author(s) 2020
}

\begin{abstract}
This paper presents a topology optimization framework for designing periodic viscoplastic microstructures under finite deformation. To demonstrate the framework, microstructures with tailored macroscopic mechanical properties, e.g., maximum viscoplastic energy absorption and prescribed zero contraction, are designed. The simulated macroscopic properties are obtained via homogenization wherein the unit cell constitutive model is based on finite strain isotropic hardening viscoplasticity. To solve the coupled equilibrium and constitutive equations, a nested Newton method is used together with an adaptive time-stepping scheme. A well-posed topology optimization problem is formulated by restriction using filtration which is implemented via a periodic version of the Helmholtz partial differential equation filter. The optimization problem is iteratively solved with the method of moving asymptotes, where the path-dependent sensitivities are derived using the adjoint method. The applicability of the framework is demonstrated by optimizing several two-dimensional continuum composites exposed to a wide range of macroscopic strains.
\end{abstract}

Keywords Topology optimization · Material design · Finite strain $\cdot$ Rate-dependent plasticity $\cdot$ Discrete adjoint sensitivity analysis

\section{Introduction}

Topology optimization is a powerful tool that enables engineers to enhance structural performance. Since the work by Bendsøe and Kikuchi N. (1988), topology optimization has rapidly evolved and been applied to design a variety of physical systems (Deaton and Grandhi 2014; Bendsoe and Sigmund 2013). Specifically, topological design methods based on the inverse homogenization approach by Sigmund (1994) have demonstrated the possibility to create novel

\begin{tabular}{l}
\hline Responsible Editor: Yoon Young Kim \\
\hline $\begin{array}{l}\text { Niklas Ivarsson } \\
\text { niklas.ivarsson@ solid.lth.se }\end{array}$ \\
Mathias Wallin \\
mathias.wallin@ solid.lth.se \\
$\quad \begin{array}{l}\text { Daniel A. Tortorelli } \\
\text { tortorelli2@ @llnl.gov }\end{array}$ \\
1 Division of Solid Mechanics, Lund University, Lund, Sweden \\
$2 \quad \begin{array}{l}\text { Center for Design and Optimization, Lawrence Livermore } \\
\text { National Laboratory, Livermore, CA, USA }\end{array}$
\end{tabular}

composite materials with enhanced properties, by optimizing the material microstructure. Examples of these include linear elastic materials with negative Poisson's ratio (Sigmund 1995), negative thermal expansion (Sigmund and Torquato 1997), and extreme bulk modulus (Huang et al. 2011), and also viscoelastic materials with extreme bulk modulus (Andreasen et al. 2014).

In the design of such architected composite materials, a common simplification in the formulation is to use linear elasticity, which implies that composite materials that exhibit irreversible processes and are exposed to large deformations cannot be designed. While linear assumptions suffice for many composite design optimization applications wherein the microstructure is subjected to moderate macroscopic strain levels, they fail to accurately model the microstructural material response when it is subjected to larger macroscopic strains. To model this microstructural response, nonlinear finite strain theory should be incorporated into the composite topology optimization.

The evaluation of the homogenized material properties becomes demanding when accounting for response nonlinearities, due to the need for iterative nonlinear analyses (Yvonnet et al. 2009). Incorporating nonlinear simulations into topology optimization problems is a formidable task. 
Even the efficient parallel programming computations, as presented in Nakshatrala et al. (2013), for the design of nonlinear elastic composites, and the model reduction technique computations, as presented in Fritzen et al. (2016), for designing multiscale elastoviscoplastic structures, remain computationally intensive tasks.

In the works by Wang et al. (2014) and Wallin and Tortorelli (2020), alternative nonlinear homogenization methods have been proposed to design architected materials. These homogenization methods use numerical tensile experiments for calculating the effective uniaxial material response and use topology optimization to achieve desired macroscopic properties. In this way, the computational effort required for the analysis is significantly reduced, since just a few desired properties are targeted.

Of the developed topology optimization methods, few consider structures exhibiting inelastic material response. Contributions incorporating small strain elastoplastic formulations include the work by Maute et al. (1998), where topology optimization is used to optimize structural ductility. Conceptual designs of energy absorbers for crashworthiness, modeled with 2D-beam elements, were established by Pedersen (2004). Protective systems with maximum energy dissipation for continuum structures subject to impact loading have also been designed by Nakshatrala and Tortorelli (2015), where a transient elastoplastic topology optimization formulation is used. Elastoplastic material modeling has further been used in Bogomolny and Amir (2012) for optimizing steel-reinforced concrete structures, in Kato et al. (2015) for optimizing composite structures for maximum energy absorption, and more recently in Wallin et al. (2016) for maximizing the energy absorbtion of structures under finite deformation. Also, in our previous paper (Ivarsson et al. 2018), we combine dynamic rate-dependent finite strain effects with topology optimization to design maximal energy absorbing structures. In the design of such energy absorbing structures, e.g., bumpers in automotive engineering, it may also be desirable to control the volume change, since devices that operate in confined spaces will be extremely stiff if the deformation is associated with a volume increase.

To date, most topology optimization frameworks that incorporate nonlinearities focus on macroscopic design; research on composite material design via inverse homogenization that incorporates inelastic constituents is scarce. Examples of such studies include the recent works by Chen et al. (2018) and Alberdi and Khandelwal (2019) which assume infinitesimal deformation. In this paper, we generate optimal topologies of periodic microstructures for maximum energy dissipation with possible prescribed zero contraction constraints under finite deformation. To do this, we incorporate the large strain isotropic hardening viscoplasticity constitutive model, proposed by Simo and Miehe (1992), into a topology optimization framework. Macroscopic nonlinear material properties are evaluated by numerical tensile and shear tests of a single unit cell that is subjected to periodic boundary conditions. Our framework builds upon the works of Ivarsson et al. (2018), as rate-dependent finite strain effects are included, but for simplicity, we neglect inertia. Contrary to our previous work, where structural design was considered, we here study microstructures optimized for maximum energy absorption. Thus, the present work allows each point in a macroscopic structure to be tailored to its specific loading rate, load path, and load magnitude it experiences. In particular, we investigate the influence of nonproportional loading and distortion constraints. Ultimately we quantify the optimization cost and constraint functions via the response from a finite strain viscoplastic boundary value problem which is solved using a nested Newton approach, wherein the momentum balance equation is coupled with local constitutive equations. We discretize the kinematics using a total Lagrangian formulation and use the backward Euler scheme to integrate the constitutive equations.

To formulate a well-posed topology optimization problem, we use restriction wherein we constrain the feature length scale in the volume fraction field by mollifying it via a periodic version of the Helmholtz partial differential equation (PDE) filter (Lazarov and Sigmund 2011). The optimization problem is solved by the method of moving asymptotes (MMA), which requires gradients to form the convex MMA approximation. For path-dependent material response, it is challenging to compute response sensitivities. This is because the sensitivities depend on the response history, as opposed to, e.g., elasticity, where they do not. To compute the gradients, we use the adjoint sensitivity analysis because the design variables in the optimization problem greatly outnumber the constraints. Several studies (Bogomolny and Amir 2012; Wallin et al. 2016; Zhang et al. 2017; Amir 2017; Ivarsson et al. 2018; Zhang and Khandelwal 2019) of topology optimization for path-dependent problems have successfully adopted the adjoint sensitivity recipe presented in Michaleris et al. (1994). This requires the evaluation and storage of the primal response trajectory, and the solution of a terminal-valued adjoint sensitivity problem. We implement this adjoint method to compute the sensitivities.

The remainder of this paper is organized as follows: In Section 2, we present the kinematics and governing mechanical balance equations. Section 3 summarizes the constitutive model and Section 4 presents the numerical solution procedure and the periodic boundary condition implementation. Section 5 describes the regularization and material interpolation schemes. This is followed by the optimization formulation and the sensitivity analysis given in Section 6. The paper is closed by presenting several 
numerical examples of optimized composite designs and drawing conclusions.

\section{Preliminaries}

For nonlinear kinematics, the current, i.e., deformed configuration $\Omega$ needs to be distinguished from the reference, i.e., undeformed configuration $\Omega_{o}$. The location of a material particle, which initially at time $t=0$ is located at $X \in \Omega_{o}$, is described by the smooth motion $\varphi$ such that its position at time $t>0$ is $\boldsymbol{x}=\boldsymbol{\varphi}(\boldsymbol{X}, t)$. The local deformation surrounding $\boldsymbol{X}$ is described by the deformation gradient $\boldsymbol{F}=\nabla_{o} \boldsymbol{\varphi}=\mathbf{1}+\nabla_{o} \boldsymbol{u}$, where $\nabla_{o}$ is the gradient with respect to the material coordinates, $\boldsymbol{X}$, and $\boldsymbol{u}=\boldsymbol{\varphi}-\boldsymbol{X}$ is the displacement field. The volumetric change is described by the Jacobian $J=\operatorname{det}(\boldsymbol{F})>0$, where the inequality ensures the mapping $\varphi$ is locally unique. The local deformation is also described by the right Cauchy-Green deformation tensor $\boldsymbol{C}=\boldsymbol{F}^{T} \boldsymbol{F}$, and the Green-Lagrange strain tensor $\boldsymbol{E}=\frac{1}{2}(\boldsymbol{C}-\mathbf{1})$. Since the constitutive model is formulated in an incremental format, we also introduce the spatial velocity gradient $\boldsymbol{l}=\dot{\boldsymbol{F}} \boldsymbol{F}^{-1}$, where $\dot{\boldsymbol{F}}=d \boldsymbol{F} / d t$.

In absence of body forces and inertia, the momentum balance laws, i.e., equilibrium equation is given by

$\nabla_{o} \cdot(\boldsymbol{F S})=\mathbf{0} \quad$ in $\Omega_{o}$

where $S$ is the symmetric second Piola-Kirchhoff stress tensor, related to the Cauchy stress $\boldsymbol{\sigma}$ via $\boldsymbol{S}=J \boldsymbol{F}^{-1} \boldsymbol{\sigma} \boldsymbol{F}^{-T}$. However, when formulating the constitutive model, we will utilize the Kirchhoff stress $\tau$ that is connected to $S$ and $\sigma$ through $\boldsymbol{\tau}=\boldsymbol{F} \boldsymbol{S} \boldsymbol{F}^{T}=J \boldsymbol{\sigma}$.

The boundary conditions associated with (1) are

$\boldsymbol{F} \boldsymbol{S} \boldsymbol{N}_{o}=\overline{\boldsymbol{t}} \quad$ on $\partial \Omega_{o t}, \quad \boldsymbol{u}=\overline{\boldsymbol{u}} \quad$ on $\partial \Omega_{o u}$,

where $N_{o}$ is the outward unit normal vector to the boundary $\partial \Omega_{o}$ of the reference body $\Omega_{o}$ and the prescribed traction $\overline{\boldsymbol{t}}$ and displacement $\overline{\boldsymbol{u}}$ are defined on the two complimentary parts of $\partial \Omega_{o}, \partial \Omega_{o t}$ and $\partial \Omega_{o u}$, respectively.

To derive the finite element formulation, we use the principal of virtual work, which can be stated as finding a smooth $\boldsymbol{u}$ that satisfies $\boldsymbol{u}=\overline{\boldsymbol{u}}$ on $\partial \Omega_{o u}$ and

$R(\boldsymbol{u}, \delta \boldsymbol{u}):=\int_{\Omega_{o}} \boldsymbol{S}: \delta \boldsymbol{E} \mathrm{d} V-\int_{\partial \Omega_{o t}} \overline{\boldsymbol{t}} \cdot \delta \boldsymbol{u} \mathrm{d} S=0$

for all smooth admissible virtual displacements $\delta \boldsymbol{u}$ that equal zero on $\partial \Omega_{o u}$. The Lagrangian virtual strain $\delta \boldsymbol{E}$, introduced in (3), is defined as $\delta \boldsymbol{E}=\frac{1}{2}\left(\boldsymbol{F}^{T} \nabla_{o} \delta \boldsymbol{u}+\right.$ $\left.\left(\nabla_{o} \delta \boldsymbol{u}\right)^{T} \boldsymbol{F}\right)$.

\section{Constitutive model}

Following Simo and Miehe (1992) and Ivarsson et al. (2018), the material model is based on isotropic hyperelasticity and isotropic hardening viscoplasticity. For finite strains, the infinitesimal deformation theory's additive split of the strain tensor is replaced by the finite deformation multiplicative split $\boldsymbol{F}=\boldsymbol{F}^{e} \boldsymbol{F}^{v p}$, where $\boldsymbol{F}^{e}$ and $\boldsymbol{F}^{v p}$ are the elastic and viscoplastic parts, respectively.

The isotropic material response is modeled in terms of the left Cauchy-Green tensor $\boldsymbol{b}^{e}=\boldsymbol{F}^{e}\left(\boldsymbol{F}^{e}\right)^{T}$, and its isochoric part, $\overline{\boldsymbol{b}}^{e}=\left(J^{e}\right)^{-2 / 3} \boldsymbol{b}^{e}$, where $J^{e}=\operatorname{det}\left(\boldsymbol{F}^{e}\right)$.

The hyperelastic response is governed by the neoHookean strain energy function (see, e.g., Simo and Hughes (1998))

$w^{e}\left(\boldsymbol{b}^{e}\right)=\frac{1}{4} \kappa\left(\left(J^{e 2}-1\right)-2 \ln \left(J^{e}\right)\right)+\frac{1}{2} \mu\left(\operatorname{tr}\left(\overline{\boldsymbol{b}}^{e}\right)-3\right)$,

where $\kappa$ and $\mu$ are the bulk and shear moduli. Under the hyperelastic assumption, the Kirchhoff stress is obtained via $\boldsymbol{\tau}=2\left(\partial w^{e} / \partial \boldsymbol{b}^{e}\right) \boldsymbol{b}^{e}$.

The rate-dependent evolution laws are obtained by maximizing the regularized dissipation

$\mathcal{D}=\dot{w}^{v p}-K \dot{\alpha}-\frac{1}{2 \eta}\langle f\rangle^{2}$

where $\dot{w}^{v p}=\boldsymbol{\tau}:\left[-\frac{1}{2} \mathcal{L} \boldsymbol{b}^{e} \boldsymbol{b}^{e-1}\right]$ is the viscoplastic power per unit volume in the reference configuration, and $\mathcal{L} \boldsymbol{b}^{e}=$ $\dot{\boldsymbol{b}}^{e}-\boldsymbol{l} \boldsymbol{b}^{e}-\boldsymbol{b}^{e} \boldsymbol{l}^{T}$ is the Lie derivative of $\boldsymbol{b}^{e}$. In (5), $\alpha$ is the internal variable that defines the isotropic hardening, $K$ defines the drag stress, $\eta \in(0, \infty)$ is the material viscosity, and the Macaulay brackets $\langle\cdot\rangle$ are defined such that $\langle x\rangle=x$ for $x \geq 0$ and $\langle x\rangle=0$ for $x<0$. The von Mises-type dynamic yield function (cf. Ristinmaa and Ottosen 2000)

$f(\boldsymbol{\tau}, K)=\left\|\boldsymbol{\tau}^{\operatorname{dev}}\right\|-\sqrt{\frac{2}{3}} \sigma_{y}(K)$

separates elastic response, $f<0$, from viscoplastic response, $f \geq 0$. In the above (6), $\tau^{d e v}$ is the deviatoric part of the Kirchhoff stress and

$\sigma_{y}(K)=\sigma_{y 0}+K$

is the current yield stress, where $\sigma_{y 0}$ is the initial yield stress. The drag stress evolves via the nonlinear exponential hardening model

$K(\alpha)=H \alpha+Y_{\infty}[1-\exp (-\delta \alpha)]$

where $H, Y_{\infty}$, and $\delta$ are the linear hardening modulus, saturation stress, and saturation exponent, respectively. 
Maximizing the regularized dissipation (5) with respect to the stresses $\tau$ and $K$ provides the evolution equations

$$
\begin{aligned}
\mathcal{L} \boldsymbol{b}^{e} & =-\frac{2}{3} \lambda \operatorname{tr}\left(\boldsymbol{b}^{e}\right) \boldsymbol{n}, \\
\dot{\alpha} & =-\lambda \frac{\partial f}{\partial K},
\end{aligned}
$$

where $\boldsymbol{n}=\boldsymbol{\tau}^{d e v} /\left\|\boldsymbol{\tau}^{d e v}\right\|$ is the normal to the yield surface, and $\lambda=\frac{1}{\eta}\langle f\rangle$ is the magnitude of the viscoplastic load increment.

The total amount of absorbed viscoplastic work, $W^{v p}$, is expressed as

$W^{v p}=\int_{0}^{T^{f}} \int_{\Omega_{o}} \dot{w}^{v p} \mathrm{~d} V \mathrm{~d} t$

where $T^{f}$ is the load duration.

\section{Numerical solution procedure}

The boundary value problem defined in (3) is spatially discretized by the finite element method in Section 4.1, and the constitutive equations are discretized with the backward Euler scheme in Section 4.2. Section 4.3 presents the imposition of the periodic boundary conditions and the nested Newton solution procedure. For further details of the finite element discretization, see Belytschko et al. (2000).

\subsection{Discretization of the equilibrium equations}

To obtain the FE-formulation, we approximate the displacement in each finite element $\Omega_{o}^{e}$ using the element shape function matrix, $\boldsymbol{N}_{e}$, according to $\boldsymbol{u}\left(\boldsymbol{X}, t_{n}\right) \approx$ $\boldsymbol{N}_{e}(\boldsymbol{X}) \hat{\boldsymbol{u}}_{e}^{(n)}$, where $\hat{\boldsymbol{u}}_{e}^{(n)}$ is the element nodal displacement vector at the discrete time step $t_{n}$. The virtual displacement is likewise interpolated as $\delta \boldsymbol{u}(\boldsymbol{X})=\boldsymbol{N}_{e}(\boldsymbol{X}) \delta \hat{\boldsymbol{u}}_{e}$, i.e., the Galerkin approach is employed. The element Lagrangian virtual strain is expressed in Voigt notation as $\delta \boldsymbol{E}_{e}\left(\boldsymbol{X}, t_{n}\right)=\boldsymbol{B}_{e}\left(\boldsymbol{X}, \hat{\boldsymbol{u}}_{e}^{(n)}\right) \delta \hat{\boldsymbol{u}}_{e}$, where the explicit expression of $\boldsymbol{B}_{e}$ is given in Wallin et al. (2018). We also use the compact internal state variable notation $\boldsymbol{w}=\left(\boldsymbol{b}^{e T} \alpha\right)^{T}$, where $\boldsymbol{b}^{e}$ and all other tensors are expressed in Voigt notation.

The residual equilibrium (3) is ultimately discretized as

$\boldsymbol{R}=\boldsymbol{F}_{\text {int }}-\boldsymbol{F}_{\text {ext }}=\mathbf{0}$.
In (12), we introduced the internal force vector $\boldsymbol{F}_{\text {int }}=\boldsymbol{F}_{\text {int }}(\hat{\boldsymbol{u}}, \boldsymbol{w})$ and the external load vector $\boldsymbol{F}_{\text {ext }}$, which are defined by

$$
\begin{aligned}
\boldsymbol{F}_{i n t} & =\mathbb{A} \int_{\Omega_{o}^{e}} \boldsymbol{B}_{e}^{T} S \mathrm{~d} V, \\
\boldsymbol{F}_{e x t} & =\xi \mathbb{A} \int_{\partial \Omega_{o t}^{e}} \boldsymbol{N}_{e}^{T} \overline{\boldsymbol{t}} \mathrm{d} S,
\end{aligned}
$$

where $\mathbb{A}$ is the finite element assembly operator, $\boldsymbol{S}=$ $\boldsymbol{S}(\hat{\boldsymbol{u}}, \boldsymbol{w})$ and $\xi \in[0,1]$ is the load parameter. For simplicity, we drop the superscript $(\cdot)^{(n)}$ and assume that all quantities are evaluated at time step $t_{n}$ unless otherwise stated.

\subsection{Integration of constitutive equations}

The residual (12) is evaluated element-wise using Gauss integration. As such, the constitutive equations need to be integrated in time at each Gauss point of each element in the mesh. To this end, the viscoplastic evolution (9) and (10) are combined with (6) and (7) and discretized in time using the fully implicit Euler scheme. Ultimately we obtain one vector and one scalar local Gauss point- $i$ residual equation. The vector equation is expressed as

$$
{ }^{1} \boldsymbol{C}_{i}=\boldsymbol{f} \boldsymbol{b}^{e,(n-1)} \boldsymbol{f}^{T}-\boldsymbol{b}^{e}-\sqrt{\frac{2}{3}}\left(\alpha-\alpha^{(n-1)}\right) \operatorname{tr}\left(\boldsymbol{b}^{e}\right) \boldsymbol{n}=\mathbf{0},
$$

where $\boldsymbol{f}=\boldsymbol{F} \boldsymbol{F}^{-1^{(n-1)}}$ is the relative deformation gradient, $\partial f / \partial K=-\sqrt{2 / 3}$ which follows from (6) and (7), and $\lambda=-\dot{\alpha} /(\partial f / \partial K)$ which follows from (9). Assuming that viscoplastic response occurs, so $\langle f\rangle=f \geq 0$ enables (10) to be expressed as $\dot{\alpha}=\frac{f}{\eta} \frac{\partial f}{\partial K}$, which gives the scalar discretized residual equation

$$
{ }^{2} C_{i}=f(\boldsymbol{\tau}, K)-\sqrt{\frac{3}{2}} \frac{\eta}{\Delta t_{n}}\left(\alpha-\alpha^{(n-1)}\right)=0 .
$$

The residuals (15) and (16) are combined as $\boldsymbol{C}_{i}=$ $\left(\begin{array}{ll}{ }^{1} C_{i}^{T} & { }^{2} C_{i}\end{array}\right)^{T}$, forming the Gauss point constitutive residual equation

$\boldsymbol{C}_{i}\left(\hat{\boldsymbol{u}}, \hat{\boldsymbol{u}}^{(n-1)}, \boldsymbol{w}, \boldsymbol{w}^{(n-1)}\right)=\mathbf{0}$.

Again, assuming $f \geq 0$, we use (6) and (9) to obtain $\dot{w}^{v p}=\left(\sqrt{\frac{2}{3}} \sigma_{y}+f\right) \frac{1}{\eta} f$ which is combined with (16) to compute the viscoplastic work of (11)

$$
\begin{aligned}
W^{v p} & =\int_{0}^{T^{f}} \int_{\Omega_{o}} \dot{w}^{v p} \mathrm{~d} V \mathrm{~d} t \\
& \left.\approx \sum_{n=1}^{N} \int_{\Omega_{o}}\left(\sigma_{y}(\alpha)+\frac{3}{2} \eta \dot{\alpha}\right)\right|_{t=t_{n}} \dot{\alpha} \Delta t_{n} \mathrm{~d} V,
\end{aligned}
$$


where the approximation $\dot{\alpha}=\left(\alpha-\alpha^{(n-1)}\right) / \Delta t_{n}$ is used. As seen here, the viscoplastic work is a function of the internal variables from all time steps, i.e., $W^{v p}=$ $W^{v p}\left(\boldsymbol{w}, \boldsymbol{w}^{(n-1)}, \ldots, \boldsymbol{w}^{(0)}\right)$.

\subsection{Periodic boundary conditions}

We now evaluate the homogenized material properties of the unit cell microstructure, i.e., composite. As part of this process, Bloch-type periodic boundary conditions are applied to a single unit cell, which implies that the displacement and traction fields on opposite boundaries are periodic, modulo a uniform term and antiperiodic, respectively. Without loss of generality, we restrict ourselves to a two-dimensional (2D) formulation.

The periodicity is based on master-slave elimination wherein the displacement on the boundaries $\partial \Omega_{o}^{x-}, \partial \Omega_{o}^{x+}$, $\partial \Omega_{o}^{y-}$, and $\partial \Omega_{o}^{y+}$ (cf. Fig. 1a) are separated into their master and slave displacements, $u_{k l}^{+}$and $u_{k l}^{-}$. Here the first subscript $k$ represents the displacement component, and the second subscript $l$ represents the boundary, e.g., $u_{x y}^{+}$ is the $u_{x}$ displacement component over the surface $\Omega_{o}^{y+}$. The differences between master and slave displacement components on opposing faces are, e.g.,

$\bar{u}_{x x}=u_{x x}^{+}-u_{x x}^{-}$,

where $\bar{u}_{x x}$ is the uniform axial displacement difference enforced across the boundaries $\partial \Omega_{o}^{x+}$ and $\partial \Omega_{o}^{x-}$, cf. Fig. $1 \mathrm{~b}$.

The slave degrees of freedom are explicitly eliminated from the nonlinear system (12) by expressing the nodal displacement vector as

$\hat{\boldsymbol{u}}=\boldsymbol{T} \check{\boldsymbol{u}}+\bar{u}_{x x} \boldsymbol{I}_{x x}+\bar{u}_{x y} \boldsymbol{I}_{x y}+\bar{u}_{y x} \boldsymbol{I}_{y x}+\bar{u}_{y y} \boldsymbol{I}_{y y}$,

where $\check{\boldsymbol{u}}$ contains the nodal displacement components of the master and the interior (belonging to $\Omega_{o} \backslash \partial \Omega_{o}$ ) degrees of freedom, and the constant matrix $\boldsymbol{T}$ defines the connection between the master and slave degrees of freedom. In (20), the vectors $\boldsymbol{I}_{x x}$ and $\boldsymbol{I}_{y x}$ have unit entries respectively for the $u_{x}$ and $u_{y}$ degrees of freedoms corresponding to the nodes on the boundary $\partial \Omega_{o}^{x+}$ and zero entries elsewhere. $\boldsymbol{I}_{x y}$ and $\boldsymbol{I}_{y y}$ are similarly defined.

For later purposes, (20) is decomposed into a dependent and independent part. To simplify the derivations, only the $x$ uniaxial load case of (19) is considered. To do this, we assign the value of $\bar{u}_{x x}$ and decompose (20) as

$\hat{\boldsymbol{u}}=\boldsymbol{P} \hat{\boldsymbol{u}}^{*}+\bar{u}_{x x} \boldsymbol{I}_{x x}$,

where $\boldsymbol{P}=\left[\begin{array}{llll}\boldsymbol{T} & \boldsymbol{I}_{x y} & \boldsymbol{I}_{y x} & \boldsymbol{I}_{y y}\end{array}\right]$, and $\hat{\boldsymbol{u}}^{*}=\left[\begin{array}{llll}\check{\boldsymbol{u}}^{T} & \bar{u}_{x y} & \bar{u}_{y x} & \bar{u}_{y y}\end{array}\right]^{T}$ contains the dependent displacements. To accommodate the added $\bar{u}_{x y}, \bar{u}_{y x}$, and $\bar{u}_{y y}$ degrees of freedom, the residuals (12) and (17) are supplemented with the null traction conditions

$\boldsymbol{I}_{x y}^{T} \boldsymbol{F}_{\text {ext }}=0, \quad \boldsymbol{I}_{y x}^{T} \boldsymbol{F}_{\text {ext }}=0, \quad \boldsymbol{I}_{y y}^{T} \boldsymbol{F}_{\text {ext }}=0$,

such that the boundaries $\partial \Omega_{o}^{y \pm}$ are macroscopically traction free and $\partial \Omega_{o}^{x \pm}$ has zero shear traction, i.e., uniaxial conditions are mimicked.

The internal virtual work $\delta W_{i n t}=\boldsymbol{F}_{i n t}^{T} \delta \hat{\boldsymbol{u}}$ together with periodicity of (21) and the null traction of (22) imply that

$$
\begin{aligned}
\delta W_{i n t}= & \boldsymbol{F}_{i n t}^{T} \boldsymbol{P} \delta \hat{\boldsymbol{u}}^{*}=\left(\boldsymbol{T}^{T} \boldsymbol{F}_{i n t}\right)^{T} \delta \check{\boldsymbol{u}}+R_{x y} \delta \bar{u}_{x y} \\
& +R_{y x} \delta \bar{u}_{y x}+R_{y y} \delta \bar{u}_{y y}=0,
\end{aligned}
$$

where, e.g., $R_{x y}=\boldsymbol{I}_{x y}^{T} \boldsymbol{F}_{i n t}$. Equation 23 is used to define the compact residual
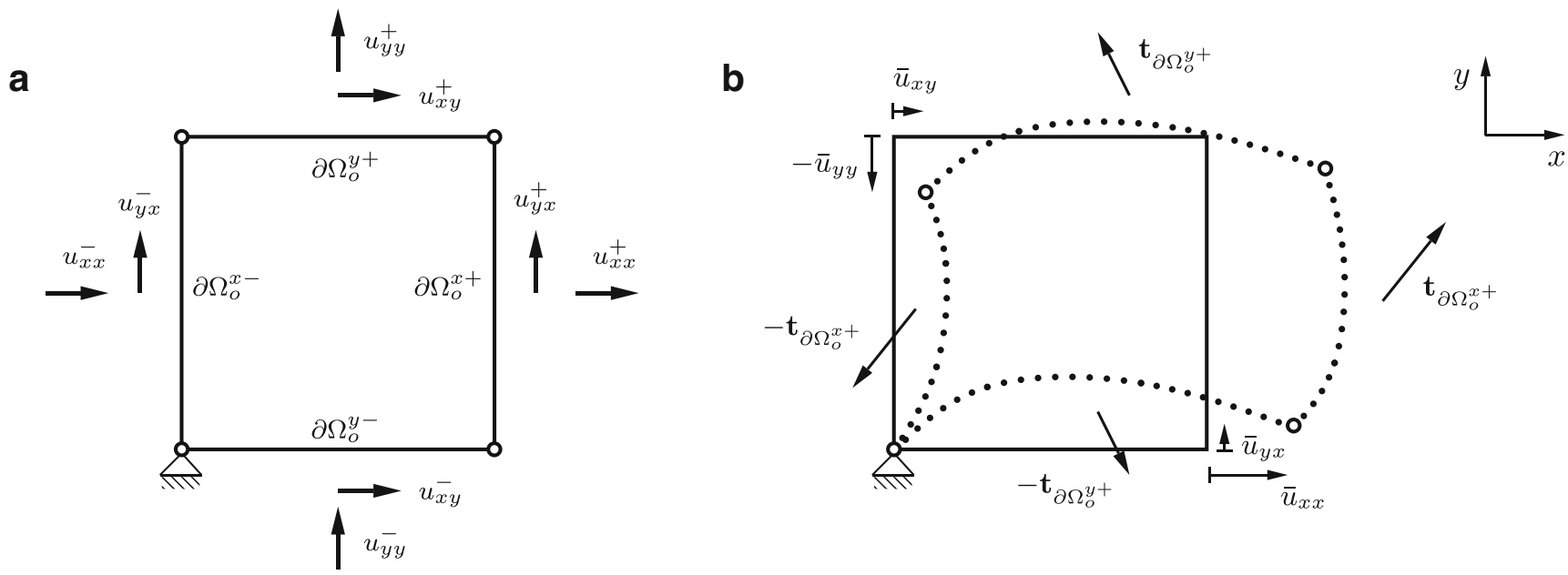

Fig. 1 Illustration of the periodic boundary conditions. a Undeformed unit cell divided into four boundaries and b deformed (dotted lines) unit cell with prescribed and unknown gap displacements, and anti-periodic traction $\mathbf{t}$ 
The coupled nonlinear system $\boldsymbol{R}^{*}=\mathbf{0}$ and $\boldsymbol{C}_{i}=$ $\mathbf{0}$ of (17) and (24) is solved using two nested Newton loops, wherein linearizations around the current $(\hat{\boldsymbol{u}}, \boldsymbol{w})$ iterate are performed using a Schur's complement approach (Ivarsson et al. 2018; Simo and Miehe 1992). At each iteration, an inner Newton loop is first completed to solve $\boldsymbol{C}_{i}\left(\hat{\boldsymbol{u}}^{(n)}, \hat{\boldsymbol{u}}^{(n-1)}, \boldsymbol{w}^{(n)}, \boldsymbol{w}^{(n-1)}\right)=\mathbf{0}$ for $\boldsymbol{w}^{(n)}$ for the given values of $\hat{\boldsymbol{u}}^{(n)}, \hat{\boldsymbol{u}}^{(n-1)}$, and $\boldsymbol{w}^{(n-1)}$. The outer loop then commences whence the linearized system

$\boldsymbol{K}_{T}^{*} d \hat{\boldsymbol{u}}^{*}=-\boldsymbol{R}^{*}$

is solved for $d \hat{\boldsymbol{u}}^{*}$, where $\boldsymbol{K}_{T}^{*}=\boldsymbol{P}^{T} \boldsymbol{K}_{T} \boldsymbol{P}$ follows from (21) and (24), in which $\boldsymbol{K}_{T}$ is the consistent tangent stiffness matrix defined by

$\boldsymbol{K}_{T}=\left[\frac{\partial \boldsymbol{F}_{i n t}}{\partial \hat{\boldsymbol{u}}}-\frac{\partial \boldsymbol{F}_{i n t}}{\partial \boldsymbol{w}}\left(\frac{\partial \boldsymbol{C}}{\partial \boldsymbol{w}}\right)^{-1} \frac{\partial \boldsymbol{C}}{\partial \hat{\boldsymbol{u}}}\right]$,

where $C$ contains the collection of the $N_{g p}$ Gauss point residuals $\boldsymbol{C}_{i}, i=1,2, \ldots, N_{g p}$. The use of the consistent tangent is crucial for preserving the quadratic rate of asymptotic convergence of the Newton iteration procedure (Simo and Taylor 1985). The successive updates of $\boldsymbol{w}^{(n)}$ and $\hat{\boldsymbol{u}}^{*(n)}$ continue until convergence is achieved. The time is then incremented and the procedure begins anew with the initial guess for the displacements $\hat{\boldsymbol{u}}_{0}^{*(n)}$. A common choice for the initial displacement guess is the previous converged solution, i.e., $\hat{\boldsymbol{u}}_{0}^{*(n)}=\hat{\boldsymbol{u}}^{*(n-1)}$. Based on our experience, this particular choice is not efficient and therefore, we use the initial guess

$\hat{\boldsymbol{u}}_{0}^{*(n)}=\frac{\xi^{(n)}}{\xi^{(n-1)}} \hat{\boldsymbol{u}}^{*(n-1)}$,

i.e., $\hat{\boldsymbol{u}}_{0}^{*(n)}$ is a scaling of the previous converged solution, which leads to

$\hat{\boldsymbol{u}}_{0}^{(n)}=\frac{\xi^{(n)}}{\xi^{(n-1)}} \boldsymbol{P} \hat{\boldsymbol{u}}^{*(n-1)}+\xi^{(n)} \bar{u}_{x x} \boldsymbol{I}_{x x}=\frac{\xi^{(n)}}{\xi^{(n-1)}} \hat{\boldsymbol{u}}^{(n-1)}$.

The first $n=1$ time step uses the initial guess $\hat{\boldsymbol{u}}_{0}^{(1)}=$ $\xi^{(1)} \bar{u}_{x x} \boldsymbol{I}_{x x}$.

Remark 1: In the numerical examples, we enforce orthotropic domain symmetry of the unit cell. For the $x$ uniaxial case, this a priori ensures $\bar{u}_{x y}=\bar{u}_{y x}=0$ and $R_{x y}=R_{y x}=0$. As such, we only enforce the $\boldsymbol{I}_{y y}^{T} \boldsymbol{F}_{\text {ext }}=0$ reaction condition to evaluate $\bar{u}_{y y}$.

Remark 2: For a pure shear load case, we prescribe $\bar{u}_{x y}>$ $0, \bar{u}_{y x}=0$ and compute $\bar{u}_{x x}$ and $\bar{u}_{y y}$ to enforce $\boldsymbol{I}_{x x}^{T} \boldsymbol{F}_{e x t}=$ 0 and $\boldsymbol{I}_{y y}^{T} \boldsymbol{F}_{\text {ext }}=0$.

\section{Regularization and material interpolation}

In this topology optimization study, we use restriction to formulate a well-posed problem. The continuous nondimensional volume fraction field $c(\boldsymbol{X})$ describes the amount of material for reference point $\boldsymbol{X}$, where locations filled with material are defined by $c(\boldsymbol{X})=1$ and void locations are identified by $c(\boldsymbol{X})=0$. The continuous volume fraction field $c$ is a function of the design field $\phi(X) \in[0,1]$ which is discretized to be piecewise uniform over the elements $\Omega_{o}^{e}$ such that $\phi(X)=\phi_{e}$ for $\boldsymbol{X} \in \Omega_{o}^{e}$. In this relationship, $c$ is obtained by smoothing $\phi$ so as to define a well-posed topology optimization problem. The smoothing uses the Helmholtz partial differential equation filtering technique, cf., e.g., Lazarov and Sigmund (2011), wherein

$-l_{o}^{2} \Delta_{o} c+c=\phi$,

where $l_{o}$ controls the length scale, i.e., smoothing and $\Delta_{o}$ is the Laplacian with respect to the material coordinates $\boldsymbol{X}$. The filtering technique has previously been used with homogeneous Neumann boundary conditions, $\nabla_{o} c \cdot \boldsymbol{N}_{o}=0$ to conserve volume. In this work, periodicity is enforced on $c$, which implies that $\nabla_{o} c \cdot \boldsymbol{N}_{o}$ is antiperiodic and hence we also conserve volume.

We solve (29) using a standard finite element formulation. The filtered continuous volume fraction field $c$ and the weight function $\delta c$ are interpolated using the element shape function vector, i.e., $c(X)={ }^{c} N_{e}(X) c_{e}$ within finite element $\Omega_{o}^{e}$, and the resulting linear system

$\boldsymbol{K}_{f} \boldsymbol{c}=\boldsymbol{F}_{f} \boldsymbol{\phi}$

is solved for $\boldsymbol{c}$, i.e., the nodal values of $c$. In (30), $\boldsymbol{K}_{f}=\mathbb{A} \int_{\Omega_{o}^{e}}\left(l_{o}^{2} \nabla_{o}{ }^{c} \boldsymbol{N}_{e}^{T} \nabla_{o}{ }^{c} \boldsymbol{N}_{e}+{ }^{c} \boldsymbol{N}_{e}^{T}{ }^{c} \boldsymbol{N}_{e}\right) \mathrm{d} V$ is the filter tangent matrix, $\boldsymbol{F}_{f}=\mathbb{A} \int_{\Omega_{o}^{e}}{ }^{c} \boldsymbol{N}_{e}^{T} \mathrm{~d} V$, and $\boldsymbol{\phi}=$ $\left[\phi_{1}, \phi_{2}, \ldots\right]^{T}$ is the design variable vector. Since the finite element discretization is fixed, the matrices $\boldsymbol{K}_{f}$ and $\boldsymbol{F}_{f}$ are computed once. In order to enforce periodicity, similar to (20), the constraint

$\boldsymbol{c}=\boldsymbol{T}_{f} \boldsymbol{c}^{*}$

is included where the connectivity matrix $\boldsymbol{T}_{f}$ connects the master nodes, stored in $\boldsymbol{c}^{*}$, to the slave nodes. Inserting (31) into (30), and premultiplying by $\boldsymbol{T}_{f}^{T}$, yields the resulting linear system

$\boldsymbol{K}_{f}^{*} c^{*}=\boldsymbol{T}_{f}^{T} \boldsymbol{F}_{f} \boldsymbol{\phi}$,

where $\boldsymbol{K}_{f}^{*}=\boldsymbol{T}_{f}^{T} \boldsymbol{K}_{f} \boldsymbol{T}_{f}$. Upon computing $\boldsymbol{c}^{*}$ from (32), the volume fraction field is obtained from (31) and the finite element interpolation $c(X)={ }^{c} N_{e}(X) c_{e}$.

To approach a distinct solid-void design, we use a Heaviside thresholding technique (Guest et al. 2004; Wang et al. 2014) and material penalization. First, the continuous 
filtered volume fraction $c$ is thresholded using the smoothed Heaviside step function $\bar{H}$, to define the physical volume fraction field

$\hat{c}=\bar{H}(c)=\frac{\tanh \left(\beta_{H} \omega\right)+\tanh \left(\beta_{H}(c-\omega)\right)}{\tanh \left(\beta_{H} \omega\right)+\tanh \left(\beta_{H}(1-\omega)\right)}$.

The parameters $\beta_{H}$ and $\omega$ are such that $\lim _{\beta_{H} \rightarrow \infty} \bar{H}(c)=$ $u_{S}(c-\omega)$, where $u_{s}$ is the unit step function.

Second, the material parameters $\kappa, \mu, H, \sigma_{y 0}$, and $Y_{\infty}$ for the constituent material are penalized via the RAMP material interpolation scheme (Stolpe and Svanberg 2001). Using the physical volume fraction $\hat{c}$ of (33), the material interpolation function for each parameter $\chi$ is defined as

$\chi\left(\hat{c} ; q, \chi_{0}\right)=\left(\delta_{0}+\frac{\hat{c}\left(1-\delta_{0}\right)}{1+q(1-\hat{c})}\right) \chi_{0}$,

where the parameter $q$ controls the level of penalization, $\chi_{0}$ is the nominal material parameter value, and $\delta_{0}=10^{-4}$ is a small residual value used in this ersatz material model to avoid numerical problems which otherwise arise if, e.g., the material stiffness is zero.

To summarize, the design variables $\phi_{e}$ are first filtered by solving (32) for $\boldsymbol{c}^{*}$, then the thresholded physical periodic volume fraction field $\hat{c}$ is computed via (31) and (33), and finally the penalized material parameters are evaluated through the application of (34) for $\chi_{0}=\{\kappa, \mu, H$, $\left.\sigma_{y 0}, Y_{\infty}\right\}$

\section{Topology optimization}

The objective considered in this topology optimization is to design a material microstructure with maximum viscoplastic energy absorption, $W^{v p}$, cf. (18), subject to displacement constraints. One displacement constraint enforced during uniaxial loading $\bar{u}_{x x}$ prescribes the transverse macroscopic displacement component $\bar{u}_{y y}$. This Poisson's ratio-type constraint is imposed by requiring

$\mathrm{g}_{\bar{u}_{y y}}=\sqrt{\frac{1}{T^{f}} \int_{0}^{T^{f}}\left(\bar{u}_{y y}-\bar{u}_{y y}^{*}\right)^{2} \mathrm{~d} t} \leq \delta_{\bar{u}}$,

and using the implicit Euler scheme to discretize (35). As seen above, $\mathrm{g}_{\bar{u}_{y y}}<\delta_{\bar{u}}$ ensures the root-mean-square deviation (RMSD) of $\bar{u}_{y y}$ from the prescribed target trajectory $\bar{u}_{y y}^{*}$ is less than a small value $\delta_{\bar{u}}$ taken as 3 . $10^{-3} \bar{u}_{x x}$. Similarly, $\bar{u}_{x x}$ can be constrained for a prescribed uniaxial loading $\bar{u}_{y y}$.

\subsection{Optimization formulation}

The topology optimization problem is to find a design $\phi$ by solving

$$
\mathcal{O}:\left\{\begin{array}{l}
\max _{\boldsymbol{\phi}} W^{v p}\left(\boldsymbol{w}^{(N)}(\hat{f}(\boldsymbol{\phi})), \ldots, \boldsymbol{w}^{(0)}(\hat{f}(\boldsymbol{\phi})), \hat{f}(\boldsymbol{\phi})\right), \\
\text { s.t. }\left\{\begin{array}{l}
g_{\bar{u}_{y y}}=\frac{1}{\delta_{\bar{u}}} \sqrt{\frac{1}{T^{f}} \sum_{n=1}^{N}\left(\bar{u}_{y y}^{(n)}-\bar{u}_{y y}^{*}\right)^{2} \Delta t_{n} \leq 1,} \\
g_{\bar{V}}=\frac{1}{\bar{V}} \int_{\Omega_{o}} \hat{c} \mathrm{~d} V \leq 1,
\end{array}\right.
\end{array}\right.
$$

where $\bar{V}$ is the maximum allowable volume in the reference configuration and the mapping

$\hat{f}(\boldsymbol{\phi})=\hat{c}\left(c\left(\boldsymbol{c}\left(\boldsymbol{c}^{*}(\boldsymbol{\phi})\right)\right)\right)$

which follows from (31)-(33) and the interpolation $c=$ ${ }^{c} \boldsymbol{N}_{e} \boldsymbol{c}_{e}$, within each element. In the above reduced space optimization problem, we treat the equilibrium (24) and local constitutive (17) residuals as implicit constraints, i.e., in (36) we require

$$
\boldsymbol{R}^{*}(\hat{\boldsymbol{u}}, \boldsymbol{w}, \hat{f}(\boldsymbol{\phi}))=\mathbf{0}, \quad \forall t_{n} \in\left[0, T^{f}\right],
$$

$\boldsymbol{C}\left(\hat{\boldsymbol{u}}, \boldsymbol{w}, \hat{\boldsymbol{u}}^{(n-1)}, \boldsymbol{w}^{(n-1)}, \hat{f}(\boldsymbol{\phi})\right)=\mathbf{0}, \quad \forall t_{n} \in\left[0, T^{f}\right]$,

where $\hat{\boldsymbol{u}}=\hat{\boldsymbol{u}}\left(\hat{\boldsymbol{u}}^{*}(\hat{f}(\boldsymbol{\phi}))\right)$ and $\boldsymbol{w}=\boldsymbol{w}(\hat{f}(\boldsymbol{\phi}))$.

Remark 3: The optimization problem (36) allows for maximization of viscoplastic energy absorption subject to a maximum volume constraint by letting $\delta_{\bar{u}} \rightarrow \infty$.

\subsection{Sensitivity analysis}

The optimization problem (36) is solved with the MMA scheme, cf. Svanberg (1987), using the standard MMA parameters as described in Svanberg (2002). The algorithm uses gradients of the objective and constraint functions to form a convex approximation of the optimization problem. We therefore perform a sensitivity analysis in each optimization iteration, and as the number of design variables greatly outnumber the constraints, we use the adjoint method presented in Michaleris et al. (1994). The sensitivity analyses for the objective function $W^{v p}$ and the constraint $g_{\bar{u}_{y y}}$ are performed in a similar manner by considering the generalized response function

$\Theta=\Theta\left(\hat{\boldsymbol{u}}^{(N)}, \ldots, \hat{\boldsymbol{u}}^{(1)}, \boldsymbol{w}^{(N)}, \ldots, \boldsymbol{w}^{(0)}, \hat{f}(\boldsymbol{\phi})\right)$. 
The sensitivities with respect to the design variable $\phi$ are obtained by multiple applications of the chain rule, i.e.,

$\frac{D \Theta}{D \phi}=\frac{D \Theta}{D \boldsymbol{c}} \frac{\partial \boldsymbol{c}}{\partial \boldsymbol{c}^{*}} \frac{\partial \boldsymbol{c}^{*}}{\partial \boldsymbol{\phi}}$

cf. (37), where $\frac{\partial \boldsymbol{c}}{\partial \boldsymbol{c}^{*}}=\boldsymbol{T}_{f}$ and $\frac{\partial \boldsymbol{c}^{*}}{\partial \boldsymbol{\phi}}=\boldsymbol{K}_{f}^{*-1}\left(\boldsymbol{T}_{f}^{T} \boldsymbol{F}_{f}\right)$ which follows from (31) and (32), respectively. In (41), we introduced $\frac{D(\cdot)}{D(\star)}$ to denote the total differentiation of $(\cdot)$ with respect to $(\star)$. Differentiation of $\Theta$ with respect to the filtered nodal volume fractions $\boldsymbol{c}$ gives

$$
\frac{D \Theta}{D \boldsymbol{c}}=\sum_{n=1}^{N}\left(\frac{\partial \Theta}{\partial \hat{\boldsymbol{u}}^{(n)}} \boldsymbol{P} \frac{D \hat{\boldsymbol{u}}^{*(n)}}{D \boldsymbol{c}}+\frac{\partial \Theta}{\partial \boldsymbol{w}^{(n)}} \frac{D \boldsymbol{w}^{(n)}}{D \boldsymbol{c}}\right)+\frac{\partial \Theta}{\partial \boldsymbol{c}},
$$

where we use the equality $\partial \hat{\boldsymbol{u}}^{(n)} / \partial \hat{\boldsymbol{u}}^{*(n)}=\boldsymbol{P}$. The implicitly defined sensitivities $D \hat{\boldsymbol{u}}^{*(n)} / D \boldsymbol{c}$ and $D \boldsymbol{w}^{(n)} / D \boldsymbol{c}$ are annihilated by augmenting a series of adjoint equations to the functional $\Theta$. To this end, the adjoint vectors $\lambda^{*(n)}$ and $\boldsymbol{\gamma}^{(n)}$ are used to define the equivalent functional

$$
\tilde{\Theta}=\Theta-\sum_{n=1}^{N} \lambda^{*(N-n+1)^{T}} \boldsymbol{R}^{*(n)}-\sum_{n=1}^{N} \boldsymbol{\gamma}^{(N-n+1)^{T}} \boldsymbol{C}^{(n)}
$$

where we emphasize that the augmented functional $\tilde{\Theta}$ equals $\Theta$, since (38) and (39) hold.

Differentiating the augmented function (43) with respect to $c$ and applying the chain rule yields

$$
\begin{aligned}
\frac{D \tilde{\Theta}}{D \boldsymbol{c}}= & \sum_{n=1}^{N}\left(\frac{\partial \Theta}{\partial \hat{\boldsymbol{u}}^{(n)}} \boldsymbol{P} \frac{D \hat{\boldsymbol{u}}^{*(n)}}{D \boldsymbol{c}}+\frac{\partial \Theta}{\partial \boldsymbol{w}^{(n)}} \frac{D \boldsymbol{w}^{(n)}}{D \boldsymbol{c}}\right)+\frac{\partial \Theta}{\partial \boldsymbol{c}} \\
& -\sum_{n=1}^{N}\left(\lambda^{*(N-n+1)}\right)^{T}\left[\boldsymbol{P}^{T} \frac{\partial \boldsymbol{F}_{i n t}^{(n)}}{\partial \hat{\boldsymbol{u}}^{(n)}} \boldsymbol{P} \frac{D \hat{\boldsymbol{u}}^{*(n)}}{D \boldsymbol{c}}\right. \\
& \left.+\boldsymbol{P}^{T} \frac{\partial \boldsymbol{F}_{i n t}^{(n)}}{\partial \boldsymbol{w}^{(n)}} \frac{D \boldsymbol{w}^{(n)}}{D \boldsymbol{c}}+\frac{\partial \boldsymbol{R}^{*(n)}}{\partial \boldsymbol{c}}\right] \\
& -\sum_{n=1}^{N}\left(\boldsymbol{\gamma}^{(N-n+1)}\right)^{T}\left[\frac{\partial \boldsymbol{C}^{(n)}}{\partial \hat{\boldsymbol{u}}^{(n)}} \boldsymbol{P} \frac{D \hat{\boldsymbol{u}}^{*(n)}}{D \boldsymbol{c}}\right. \\
& +\frac{\partial \boldsymbol{C}^{(n)}}{\partial \boldsymbol{w}^{(n)}} \frac{D \boldsymbol{w}^{(n)}}{D \boldsymbol{c}}+\frac{\partial \boldsymbol{C}^{(n)}}{\partial \hat{\boldsymbol{u}}^{(n-1)}} \boldsymbol{P} \frac{D \hat{\boldsymbol{u}}^{*(n-1)}}{D \boldsymbol{c}} \\
& \left.+\frac{\partial \boldsymbol{C}^{(n)}}{\partial \boldsymbol{w}^{(n-1)}} \frac{D \boldsymbol{w}^{(n-1)}}{D \boldsymbol{c}}+\frac{\partial \boldsymbol{C}^{(n)}}{\partial \boldsymbol{c}}\right] .
\end{aligned}
$$

The above is divided into an explicit, $D \tilde{\Theta}_{E} / D \boldsymbol{c}$, and implicit, $D \tilde{\Theta}_{I} / D \boldsymbol{c}$, part as $D \tilde{\Theta} / D \boldsymbol{c}=D \tilde{\Theta}_{E} / D \boldsymbol{c}+$ $D \tilde{\Theta}_{I} / D \boldsymbol{c}$, where the explicit part is

$$
\begin{aligned}
\frac{D \tilde{\Theta}_{E}}{D \boldsymbol{c}}= & \frac{\partial \Theta}{\partial \boldsymbol{c}}-\sum_{n=1}^{N}\left(\lambda^{*(N-n+1)}\right)^{T} \frac{\partial \boldsymbol{R}^{*(n)}}{\partial \boldsymbol{c}} \\
& -\sum_{n=1}^{N}\left(\boldsymbol{\gamma}^{(N-n+1)}\right)^{T} \frac{\partial \boldsymbol{C}^{(n)}}{\partial \boldsymbol{c}}
\end{aligned}
$$

and (the transpose of) the implicit part is

$$
\begin{aligned}
\left(\frac{D \tilde{\Theta}_{I}}{D \boldsymbol{c}}\right)^{T}= & -\sum_{n=1}^{N-1}\left(\frac{D \hat{\boldsymbol{u}}^{*(n)}}{D \boldsymbol{c}}\right)^{T} \boldsymbol{P}^{T}\left[-\left(\frac{\partial \Theta}{\partial \hat{\boldsymbol{u}}^{(n)}}\right)^{T}\right. \\
& +\left(\frac{\partial \boldsymbol{F}_{i n t}^{(n)}}{\partial \hat{\boldsymbol{u}}^{(n)}}\right)^{T} \boldsymbol{P} \boldsymbol{\lambda}^{*(N-n+1)} \\
& +\left(\frac{\partial \boldsymbol{C}^{(n)}}{\partial \hat{\boldsymbol{u}}^{(n)}}\right)^{T} \boldsymbol{\gamma}^{(N-n+1)} \\
& +\left(\frac{\partial \boldsymbol{C}^{(n+1)}}{\partial \hat{\boldsymbol{u}}^{(n)}}\right)^{T} \boldsymbol{\gamma}^{(N-n)}-\sum_{n=1}^{N-1}\left(\frac{D \boldsymbol{w}^{(n)}}{D \boldsymbol{c}}\right)^{T} \\
& {\left[-\left(\frac{\partial \Theta}{\partial \boldsymbol{w}^{(n)}}\right)^{T}+\left(\frac{\partial \boldsymbol{F}_{i n t}^{(n)}}{\partial \boldsymbol{w}^{(n)}}\right)^{T} \boldsymbol{P} \boldsymbol{\lambda}^{*(N-n+1)}\right.} \\
& +\left(\frac{\partial \boldsymbol{C}^{(n)}}{\partial \boldsymbol{w}^{(n)}}\right)^{T} \boldsymbol{\gamma}^{(N-n+1)} \\
& \left.+\left(\frac{\partial \boldsymbol{C}^{(n+1)}}{\partial \boldsymbol{w}^{(n)}}\right)^{T} \boldsymbol{\gamma}^{(N-n)}\right] \\
& +\left(\frac{D \hat{\boldsymbol{u}}^{*(N)}}{D \boldsymbol{c}}\right)^{T} \boldsymbol{P}^{T}\left[-\left(\frac{\partial \Theta}{\partial \hat{\boldsymbol{u}}^{(N)}}\right)^{T}\right. \\
& \left.+\left(\frac{\partial \boldsymbol{F}_{i n t}^{(N)}}{\partial \hat{\boldsymbol{u}}^{(N)}}\right)^{T} \boldsymbol{P} \boldsymbol{\lambda}^{*(1)}+\left(\frac{\partial \boldsymbol{C}^{(N)}}{\partial \boldsymbol{w}^{(N)}}\right)^{T} \boldsymbol{P} \boldsymbol{\gamma}^{*(1)}+\left(\frac{\partial \boldsymbol{C}^{(N)}}{\partial \hat{\boldsymbol{u}}^{(N)}}\right)^{T} \boldsymbol{\gamma}^{(1)}\right] \\
& \left.\left.+\frac{\partial \Theta}{\partial \boldsymbol{w}^{(N)}}\right)^{T}\right]
\end{aligned}
$$

The implicitly defined derivatives $D \hat{\boldsymbol{u}}^{*(N)} / D \boldsymbol{c}$ and $D \boldsymbol{w}^{(N)} / D \boldsymbol{c}$ in (46) are annihilated by making judicious 
choices of the adjoint vectors. We first require that $\lambda^{(1)}$ and $\boldsymbol{\gamma}^{(1)}$ satisfy

$$
\begin{aligned}
& \boldsymbol{P}^{T}\left[\left(\frac{\partial \boldsymbol{F}_{i n t}^{(N)}}{\partial \hat{\boldsymbol{u}}^{(N)}}\right)^{T} \boldsymbol{P} \boldsymbol{\lambda}^{*(1)}+\left(\frac{\partial \boldsymbol{C}^{(N)}}{\partial \hat{\boldsymbol{u}}^{(N)}}\right)^{T} \boldsymbol{\gamma}^{(1)}\right] \\
= & \boldsymbol{P}^{T}\left(\frac{\partial \Theta}{\partial \hat{\boldsymbol{u}}^{(N)}}\right)^{T}, \\
& \left(\frac{\partial \boldsymbol{F}_{\text {int }}^{(N)}}{\partial \boldsymbol{w}^{(N)}}\right)^{T} \boldsymbol{P} \lambda^{*(1)}+\left(\frac{\partial \boldsymbol{C}^{(N)}}{\partial \boldsymbol{w}^{(N)}}\right)^{T} \boldsymbol{\gamma}^{(1)}=\left(\frac{\partial \Theta}{\partial \boldsymbol{w}^{(N)}}\right)^{T} .
\end{aligned}
$$

Or, by inserting (48) into (47) and some rearrangement, satisfy

$$
\begin{aligned}
\left(\boldsymbol{K}_{T}^{*(N)}\right)^{T} \lambda^{*(1)}= & \boldsymbol{P}^{T}\left[\left(\frac{\partial \Theta}{\partial \hat{\boldsymbol{u}}^{(N)}}\right)^{T}-\left(\frac{\partial \boldsymbol{C}^{(N)}}{\partial \hat{\boldsymbol{u}}^{(N)}}\right)^{T}\right. \\
& \left.\times\left(\frac{\partial \boldsymbol{C}^{(N)}}{\partial \boldsymbol{w}^{(N)}}\right)^{-T}\left(\frac{\partial \Theta}{\partial \boldsymbol{w}^{(N)}}\right)^{T}\right], \\
\left(\frac{\partial \boldsymbol{C}^{(N)}}{\partial \boldsymbol{w}^{(N)}}\right)^{T} \boldsymbol{\gamma}^{(1)}= & {\left[\left(\frac{\partial \Theta}{\partial \boldsymbol{w}^{(N)}}\right)^{T}-\left(\frac{\partial \boldsymbol{F}_{\text {int }}^{(N)}}{\partial \boldsymbol{w}^{(N)}}\right)^{T} \boldsymbol{P} \lambda^{*(1)}\right], }
\end{aligned}
$$

where $\boldsymbol{K}_{T}^{*(N)}=\boldsymbol{P}^{T} \boldsymbol{K}_{T}^{(N)} \boldsymbol{P}$ is the tangent operator at the terminal time step $t_{N}$. Once $\lambda^{*(1)}$ is obtained from (49), the adjoint variable $\boldsymbol{\gamma}^{(1)}$ is obtained from (50). In the remaining time steps of the adjoint analysis, we determine the $\lambda^{*(n)}$ and $\boldsymbol{\gamma}^{(n)}$ to annihilate $\frac{D \boldsymbol{w}^{(N-n+1)}}{D \boldsymbol{c}}$ and $\frac{D \hat{\boldsymbol{u}}^{*(N-n+1)}}{D \boldsymbol{c}}$ for $n=$ $2, \ldots, N$, by solving

$$
\begin{aligned}
& \boldsymbol{P}^{T}\left[\left(\frac{\partial \boldsymbol{F}_{i n t}^{(N-n+1)}}{\partial \hat{\boldsymbol{u}}^{(N-n+1)}}\right)^{T} \boldsymbol{P} \boldsymbol{\lambda}^{*(n)}+\left(\frac{\partial \boldsymbol{C}^{(N-n+1)}}{\partial \hat{\boldsymbol{u}}^{(N-n+1)}}\right)^{T} \boldsymbol{\gamma}^{(n)}\right] \\
= & \boldsymbol{P}^{T}\left(\frac{\partial \Theta}{\partial \hat{\boldsymbol{u}}^{(N-n+1)}}\right)^{T}-\boldsymbol{P}^{T}\left(\frac{\partial \boldsymbol{C}^{(N-n+2)}}{\partial \hat{\boldsymbol{u}}^{(N-n+1)}}\right)^{T} \boldsymbol{\gamma}^{(n-1)}(51) \\
& \left(\frac{\partial \boldsymbol{F}_{i n t}^{(N-n+1)}}{\partial \boldsymbol{w}^{(N-n+1)}}\right)^{T} \boldsymbol{P} \boldsymbol{\lambda}^{*(n)}+\left(\frac{\partial \boldsymbol{C}^{(N-n+1)}}{\partial \boldsymbol{w}^{(N-n+1)}}\right)^{T} \boldsymbol{\gamma}^{(n)} \\
= & {\left[\left(\frac{\partial \Theta}{\partial \boldsymbol{w}^{(N-n+1)}}\right)^{T}-\left(\frac{\partial \boldsymbol{C}^{(N-n+2)}}{\partial \boldsymbol{w}^{(N-n+1)}}\right)^{T} \boldsymbol{\gamma}^{(n-1)}\right] . }
\end{aligned}
$$

Alternatively, substituting (52) into (51), rearranging and using (26), we solve

$$
\begin{aligned}
& \left(\boldsymbol{K}_{T}^{*(N-n+1)}\right)^{T} \lambda^{*(n)}=-\boldsymbol{\Gamma}^{*(N-n+1)} \\
& \left(\frac{\partial \boldsymbol{C}^{(N-n+1)}}{\partial \boldsymbol{w}^{(N-n+1)}}\right)^{T} \boldsymbol{\gamma}^{(n)}=\left[\left(\frac{\partial \Theta}{\partial \boldsymbol{w}^{(N-n+1)}}\right)^{T}\right. \\
& \left.-\left(\frac{\partial \boldsymbol{C}^{(N-n+2)}}{\partial \boldsymbol{w}^{(N-n+1)}}\right)^{T} \boldsymbol{\gamma}^{(n-1)}-\left(\frac{\partial \boldsymbol{F}_{i n t}^{(N-n+1)}}{\partial \boldsymbol{w}^{(N-n+1)}}\right)^{T} \boldsymbol{P} \lambda^{*(n)}\right]
\end{aligned}
$$

where

$$
\begin{aligned}
\boldsymbol{\Gamma}^{*(N-n+1)}= & \boldsymbol{P}^{T}\left\{\left(\frac{\partial \boldsymbol{C}^{(N-n+1)}}{\partial \hat{\boldsymbol{u}}^{(N-n+1)}}\right)^{T}\left(\frac{\partial \boldsymbol{C}^{(N-n+1)}}{\partial \boldsymbol{w}^{(N-n+1)}}\right)^{-T}\right. \\
& \times\left[\left(\frac{\partial \Theta}{\partial \boldsymbol{w}^{(N-n+1)}}\right)^{T}-\left(\frac{\partial \boldsymbol{C}^{(N-n+2)}}{\partial \boldsymbol{w}^{(N-n+1)}}\right)^{T}\right. \\
& \left.\times \boldsymbol{\gamma}^{(n-1)}\right]-\left(\frac{\partial \Theta}{\partial \hat{\boldsymbol{u}}^{(N-n+1)}}\right)^{T} \\
& \left.+\left(\frac{\partial \boldsymbol{C}^{(N-n+2)}}{\partial \hat{\boldsymbol{u}}^{(N-n+1)}}\right)^{T} \boldsymbol{\gamma}^{(n-1)}\right\}
\end{aligned}
$$

is the adjoint load vector at step $t_{N-n+1}$. Equation 53 is solved for $\lambda^{*(n)}$ and subsequently used in (54) to obtain $\boldsymbol{\gamma}^{(n)}$.

Once the adjoint vectors $\lambda^{*(n)}$ and $\boldsymbol{\gamma}^{(n)}$ are computed, the sensitivity $D \Theta / D c=D \tilde{\Theta}_{E} / D c$ is obtained from (45), and $D \Theta / D \phi$ is computed via (41).

The sensitivity of the constraint $g_{\bar{u}_{y y}}$ is found from the above derivations by equating $\Theta=g_{\bar{u}_{y y}}$ and thus $\partial \Theta / \partial \boldsymbol{c}=$ $\mathbf{0}, \partial \Theta / \partial \boldsymbol{w}^{(n)}=\mathbf{0}$, and

$\frac{\partial \Theta}{\partial \hat{\boldsymbol{u}}^{(n)}}=\frac{1}{\delta_{\bar{u}} T^{f}}\left(\delta_{\bar{u}} g_{\bar{u}_{y y}}\right)^{-1 / 2}\left(\bar{u}_{y y}^{(n)}-\bar{u}_{y y}^{*}\right) \Delta t_{n} \mathbf{1}_{y y}^{T}$,

where we used $\bar{u}_{y y}^{(n)}=\mathbf{1}_{y y}^{T} \hat{\boldsymbol{u}}^{(n)}$, where the vector $\mathbf{1}_{y y}$ has a single unit entry corresponding to the $\bar{u}_{y y}$ degree of freedom of the unit cell and is zero elsewhere, cf. the discussion following (21).

For the objective function, $\Theta=W^{v p}$, the sensitivity is found by using

$$
\begin{aligned}
\frac{\partial \Theta}{\partial \boldsymbol{c}}= & \sum_{n=1}^{N} \mathbb{A} \int_{\Omega_{o}^{e}}\left(\chi_{\sigma_{y 0}}^{\prime}+\chi_{H}^{\prime} \alpha\right. \\
& \left.+\chi_{Y_{\infty}}^{\prime}[1-\exp (-\delta \alpha)]\right)\left.\right|_{t=t_{n}} \dot{\alpha} \Delta t_{n}{ }^{c} N_{e}^{T} \bar{H}^{\prime} \mathrm{d} V
\end{aligned}
$$

which follows from (7), (8), (18) and (34), and where e.g., $\chi_{\sigma_{y 0}}=\chi\left(\hat{c} ; q, \sigma_{y 0}\right)$. In (57), we also used $\partial \hat{c} / \partial c=\bar{H}^{\prime}$ and 
$\partial c / \partial \boldsymbol{c}={ }^{c} N_{e}^{T}$ within the element $\Omega_{o}^{e}$. For the $\partial \Theta / \partial \boldsymbol{w}^{(n)}$ computation, $\partial \Theta / \partial \overline{\boldsymbol{b}}^{e(n)},=\mathbf{0}, n=1, \ldots, N$ and

$$
\begin{aligned}
\frac{\partial \Theta}{\partial \alpha^{(n)}}= & \left.\sum_{n=1}^{N} \int_{\Omega_{o}}\left(\frac{\partial K}{\partial \alpha}+\frac{3}{2} \frac{\eta}{\Delta t_{n}}\right)\right|_{t=t_{n}} \dot{\alpha} \Delta t_{n} \mathrm{~d} V \\
& +\left.\sum_{n=1}^{N} \int_{\Omega_{o}}\left(\chi_{\sigma_{y}(\alpha)}+\frac{3}{2} \eta \dot{\alpha}\right)\right|_{t=t_{n}} \mathrm{~d} V,
\end{aligned}
$$

where $\partial K / \partial \alpha=\chi_{H}+\chi_{Y_{\infty}} \delta \exp (-\delta \alpha)$ and $\chi_{\sigma_{y}(\alpha)}=$ $\chi_{\sigma_{y 0}}+\chi_{H} \alpha+\chi_{Y_{\infty}}[1-\exp (-\delta \alpha)]$. Finally, we note that $\partial \Theta / \partial \hat{\boldsymbol{u}}^{(n)}=\mathbf{0}$.

The last two terms of the sensitivity $D \tilde{\Theta}_{E} / D \boldsymbol{c}$ of (45) are computed in the same manner for both the constraint $\Theta=g_{\bar{u}_{y y}}$ and for the objective function $\Theta=W^{v p}$. Using (13), (24), $\partial \hat{c} / \partial c=\bar{H}^{\prime}$ and $\partial c / \partial c={ }^{c} N_{e}^{T}$ within $\Omega_{o}^{e}$, the first term of (45) is expressed as

$$
\begin{aligned}
& \sum_{n=1}^{N}\left(\lambda^{*(N-n+1}\right)^{T} \frac{\partial \boldsymbol{R}^{*(n)}}{\partial \boldsymbol{c}} \\
= & \sum_{n=1}^{N} \mathbb{A} \int_{\Omega_{o}^{e}}{ }^{c} \boldsymbol{N}_{e}^{T}\left(\lambda_{e}^{(N-n+1)^{T}} \boldsymbol{B}_{e}^{(n)} \frac{\partial \boldsymbol{S}^{(n)}}{\partial \hat{c}} \bar{H}^{\prime}\right) \mathrm{d} V
\end{aligned}
$$

where $\lambda_{e}$ contains the $\Omega_{o}^{e}$ element components of $\lambda=\boldsymbol{P} \lambda^{*}$, and $\partial \boldsymbol{S}^{(n)} / \partial \hat{c}=\boldsymbol{F}^{-1}\left(\partial \boldsymbol{\tau}^{(n)} / \partial \hat{c}\right) \boldsymbol{F}^{-T}$ where

$\partial \boldsymbol{\tau} / \partial \hat{c}=\frac{\chi_{\kappa}^{\prime}}{2}\left(\left(J^{e}\right)^{2}-1\right) \mathbf{1}+\chi_{\mu}^{\prime} \overline{\boldsymbol{b}}^{e, d e v}$

which follows from (4), $\boldsymbol{\tau}=2\left(\partial w^{e} / \partial \boldsymbol{b}^{e}\right) \boldsymbol{b}^{e}, \boldsymbol{\tau}^{\text {dev }}=$ $\mu \overline{\boldsymbol{b}}^{e, d e v}$ and (34). The last term of the sensitivity (45) is computed via

$$
\begin{aligned}
& \sum_{n=1}^{N}\left(\boldsymbol{\gamma}^{(N-n+1)}\right)^{T} \frac{\partial \boldsymbol{C}^{(n)}}{\partial \boldsymbol{c}} \\
= & \sum_{n=1}^{N} \mathbb{A}^{\boldsymbol{c}} \boldsymbol{N}_{e}^{T}\left(\sum_{i=1}^{N_{g p}} \boldsymbol{\gamma}_{i}^{(N-n+1)^{T}} \frac{\partial \boldsymbol{C}_{i}^{(n)}}{\partial \hat{c}}\right) \bar{H}^{\prime},
\end{aligned}
$$

where $\gamma_{i}$ contains the Gauss point $i$ and element $\Omega_{o}^{e}$ components of $\boldsymbol{\gamma}$, and $\partial \boldsymbol{C}_{i} / \partial \hat{c}=\left(\begin{array}{ll}\mathbf{0}^{T} & \partial{ }^{2} C_{i} / \partial \hat{c}\end{array}\right)^{T}$, where $\partial^{2} C_{i} / \partial \hat{c}=\chi_{\mu}^{\prime}\left\|\bar{b}^{e, d e v}\right\|+\sqrt{2 / 3}\left(\partial \chi_{\sigma_{y(\alpha)}} / \partial \hat{c}\right)$ which follows from (6), $\boldsymbol{\tau}^{d e v}=\mu \overline{\boldsymbol{b}}^{e, d e v}$, (16) and (34).

\section{Numerical examples}

In this section, the presented topology optimization framework is applied to design several solid-void composite materials for maximal energy absorption. The material parameters for the full material are those of steel and are presented in Ivarsson et al. (2018).
The primal viscoplasticity problem is solved with an adaptive time stepping scheme such that

$\Delta t_{n+1}=\Delta t_{n}\left(f_{\text {iter }} / N_{\text {newt }}\right)^{v}$,

where $N_{\text {newt }}$ is the number of Newton iterations that is required to attain convergence at the current time step $t_{n}$, cf., e.g., Borgqvist and Wallin (2013), and the parameters $f_{i t e r}$ and $v$ are chosen as $f_{\text {iter }}=4$ and $v=0.4$. The convergence criterion is such that $\left|\boldsymbol{R}^{* T} \Delta \hat{\boldsymbol{u}}^{*}\right|<10^{-10}$ $\mathrm{Nmm}$ and diverging time steps are restarted with $\Delta t_{n+1} \leftarrow$ $\Delta t_{n+1} / 2$. The initial time increment is $\Delta t_{1}=T^{f} / 500$ and the time increments used to perform the sensitivity analysis are consistent with those used to solve the primal problem.

Although the analysis is for two dimensions, we use fully integrated 8-node tri-linear brick elements to discretize the $1 \mathrm{~mm} \times 1 \mathrm{~mm} \times 0.01 \mathrm{~mm}$ unit cell with $80 \times 80 \times 1$ elements. Plane strain boundary conditions are enforced on the $z$-displacement components. Because of this plane strain condition, the $z$-faces of the designs are necessarily periodic.

The Heaviside threshold parameter is $\omega=0.5$ and a continuation strategy is used for $\beta_{H}$ wherein its initial value $\beta_{o}=10^{-10}$ is used for the first 80 MMA optimization iterations, whereafter it is increased by increments of 1 every $n_{\beta}$ design updates until the maximum value of $\beta_{H}=$ 10 is reached. In the first two examples $n_{\beta}=10$, and for the optimizations including displacement constraints, the continuation is slower with $n_{\beta}=20$. The RAMP penalization parameter is $q=10$ for $\kappa, \mu, H$, and $Y_{\infty}$. To avoid large viscoplastic strain in low-density elements, the penalization parameter for the initial flow stress $\sigma_{y 0}$ is chosen such that $q_{\sigma_{y 0}}=2<q$. The maximum volume fraction is $\bar{V}=0.4$ and the filter length scale parameter is $l_{o}=1.25 / 80 \mathrm{~mm}$.

In this study, 4-fold orthotropic domain symmetry of the design is imposed (case $a$ ), cf. Fig. 2a. This greatly reduces the number of design variables. Moreover, in the analysis, e.g., for uniaxial loading (1) the periodic boundary conditions are replaced by the usual domain boundary conditions over the quarter domain model and (2) zero shear forces are ensured (see, e.g., Wallin and Tortorelli (2020)), i.e., we necessarily have $R_{x y}=R_{y x}=0$. Thus, we save computational effort as we need not consider the $\boldsymbol{I}_{x y}^{T} \boldsymbol{F}_{e x t}=0$ and $\boldsymbol{I}_{y x}^{T} \boldsymbol{F}_{e x t}=0$ equations. We also impose cubic 8 -fold domain symmetry (case $b$ ), cf. Fig. 2b. This further reduces the number of design variables; however, to avoid the imposition of complicated boundary constraints, we analyze this as if it has 4-fold symmetry. The latter case $b$ ensures microstructures that perform identically when subjected to uniaxial loads $\bar{u}_{x x}$ and $\bar{u}_{y y}$ of same magnitude and thus, only one uniaxial load case is simulated. 
a
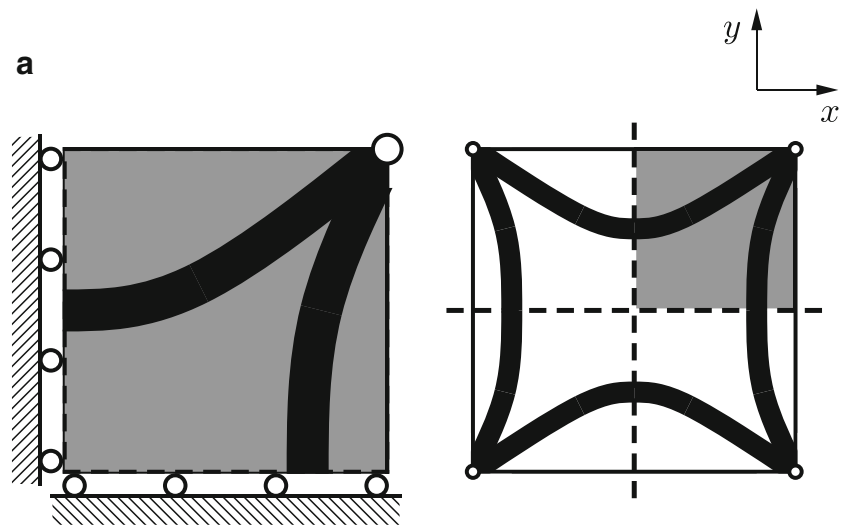

b
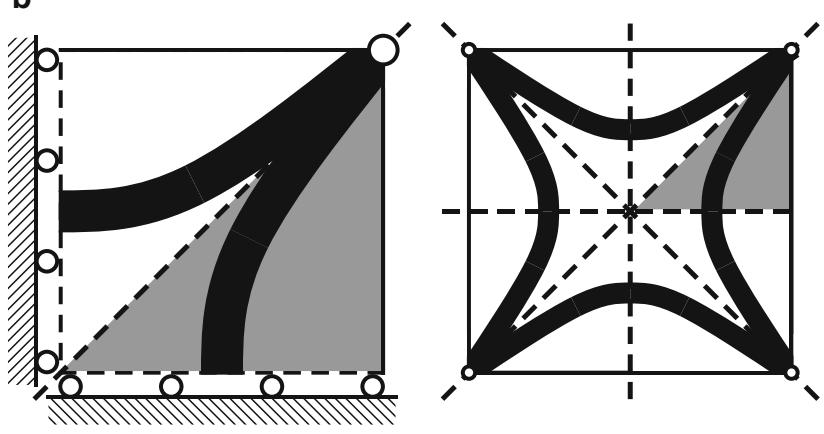

Fig. 2 Illustration of symmetry boundary conditions for domain with a 4-fold symmetry and b with 8-fold symmetry

The 4-fold symmetry also simplifies the PDE filter analysis as periodic boundary conditions are replaced by zero flux conditions on all faces.

The initial design must be nonuniform to avoid initially uniform sensitivities that inhibit the optimization (Alberdi and Khandelwal 2019; Träff et al. 2019). The optimization algorithm is therefore initiated such that all design variables are set to $\phi_{e}=0.4$, followed by a perturbation $\delta$. We consider two different perturbations; (a) a value $\delta_{4}$ is added to the four center elements, i.e., $\phi_{e}=0.4+\delta_{4}$, and (b) a different random number perturbation is added to all elements, ${ }^{1}$ such that $\phi_{e}=0.4+\delta_{r n d} \times(\operatorname{rand}(0,1)-0.5)$.

\subsection{Constitutive model}

Prior to the optimization, we demonstrate the rate effects associated with the constitutive model. The demonstration is performed using a single element model of volume fraction field $\phi_{e}=1$, by simulating the uniaxial response for $\bar{u}_{x x}=0.05 \mathrm{~mm}$ with symmetry boundary conditions of Fig. 2a. Figure 3 shows the macroscopic force component $\boldsymbol{I}_{x x}^{T} \boldsymbol{F}_{i n t}=F_{x x}$, normalized by a factor of $1 /\left(\sigma_{y 0} A_{o}\right)$, where $A_{o}=0.01 \mathrm{~mm}^{2}$ is the area of the loaded unit cell

\footnotetext{
${ }^{1}$ We use Fortrans' "RANDOM_NUMBER," based on George Marsaglia's KISS (Keep It Simple, Stupid).
}

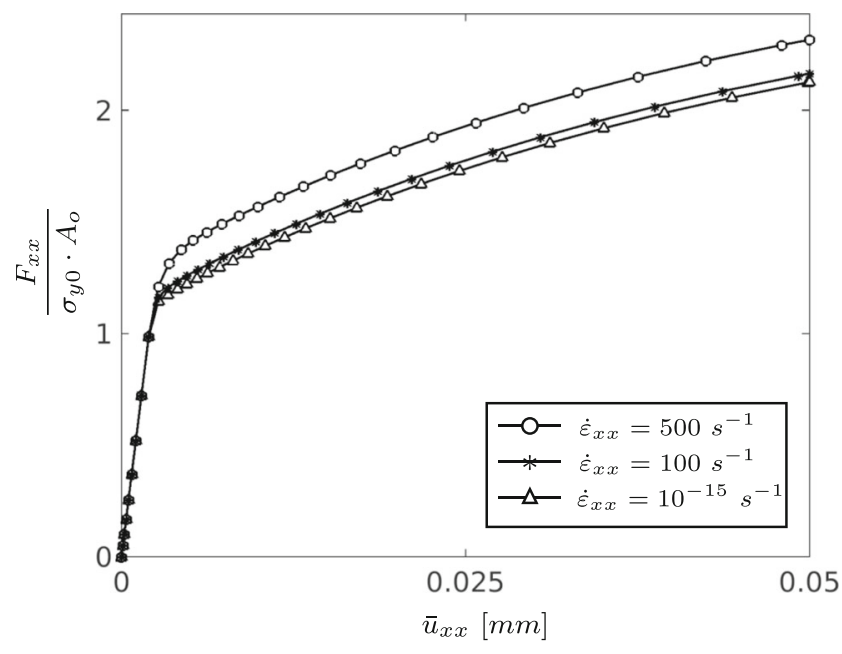

Fig. 3 Macroscopic normalized forces $F_{x x} /\left(\sigma_{y 0} A_{o}\right)$ versus load $\bar{u}_{x x}$ for the one element model

$x$-face in the reference configuration, versus load $\bar{u}_{x x}$ for the three different macroscopic engineering strain rates $\dot{\varepsilon}_{x x}=$ $10^{-15} s^{-1}, \dot{\varepsilon}_{x x}=100 s^{-1}$, and $\dot{\varepsilon}_{x x}=500 s^{-1}$. The figure clearly shows the influence of the strain rate effects on the microstructural response.

\subsection{Test problem: two simple load cases}

We start by designing materials subjected to a slow loading rate $\dot{\varepsilon}=10^{-15} s^{-1}$, without any displacement constraints. We do not enforce domain symmetry in this initial example and we consider two load cases: ( $a$ ) a uniaxial tensile load case where $\bar{u}_{x x}=0.01 \mathrm{~mm}$ is prescribed and we solve for $\bar{u}_{x y}, \bar{u}_{y x}, \bar{u}_{y y}$, and (b) a pure shear load case where $\bar{u}_{x y}=$ $0.01 \mathrm{~mm}$ and $\bar{u}_{y x}=0 \mathrm{~mm}$ are prescribed and we solve for $\bar{u}_{x x}$ and $\bar{u}_{y y}$. Figure 4 shows two optimized microstructural designs, both initiated with $\delta_{4}=0.6$, i.e., the four center elements are assigned the value 1 . The yellow and blue regions represent the base steel material and void, respectively, and we emphasize that the physical volume fraction field $\hat{c}$ is plotted. As expected, the two structures dissipating maximum energy consist of bars aligned with the maximum principal stress axes. Clearly, these designs are periodic, and although not explicitly enforced, they respectively exhibit 4-fold and 8-fold domain symmetries.

\subsection{Biaxial tensile loads}

In many practical applications, it is of great importance that structures can withstand more complex load cases than those of uniaxial tension and pure shear. Therefore, we consider a biaxial loading scenario, $\bar{u}_{x x}=\bar{u}_{y y}=0.01 \mathrm{~mm}$ again with $\dot{\varepsilon}=10^{-15} s^{-1}$, cf. Fig. 5. In this study, we use 4-fold domain symmetry and the corresponding symmetry boundary conditions of Fig. 2 a. 


\section{a $\quad \bar{u}_{x x}=0.01 \mathrm{~mm}$}

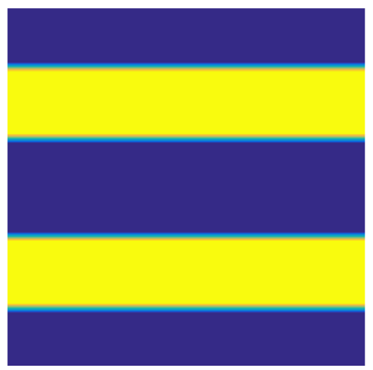

b $\quad \bar{u}_{x y}=0.01 \mathrm{~mm}$
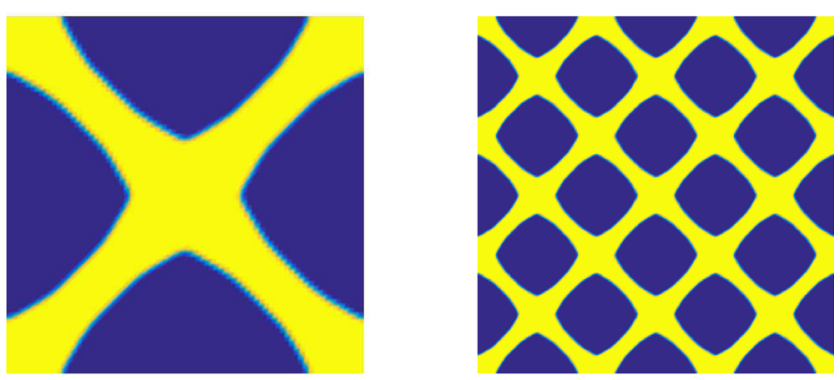

Fig. 4 Undeformed optimized unit cells (left) and array of $3 \times 3$ unit cells (right)

We present optimized microstructures obtained using three different initial designs. Figure $6 \mathrm{a}$ and $\mathrm{b}$ show two designs optimized with $\delta_{4}=0.1$ and $\delta_{4}=-0.1$, respectively. The topology of Design 6a consists of perpendicular bars aligned in the loading directions, whereas Design $6 \mathrm{~b}$ has a more complex microstructure with both rectangular and elliptical material regions. This difference is reflected in the performance as Design 6a absorbs $1.0 \%$ more viscoplastic energy than Design 6b, (cf. Fig. 7), i.e., Design 6b is a local maxima. We also note that Design 6 a absorbs approximately

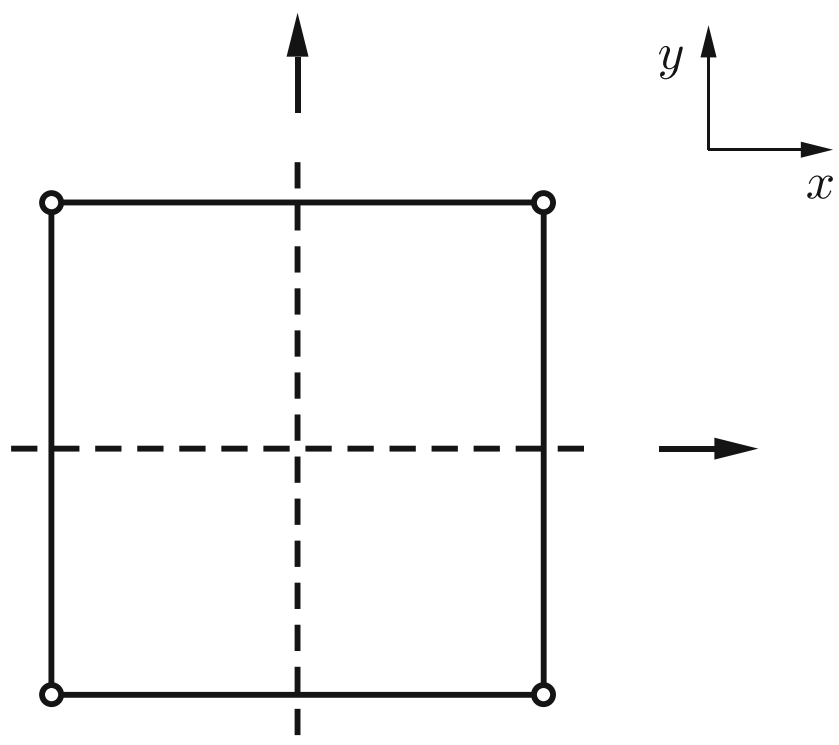

Fig. 5 Illustration of the biaxial load case a $\quad \delta_{4}=0.1$
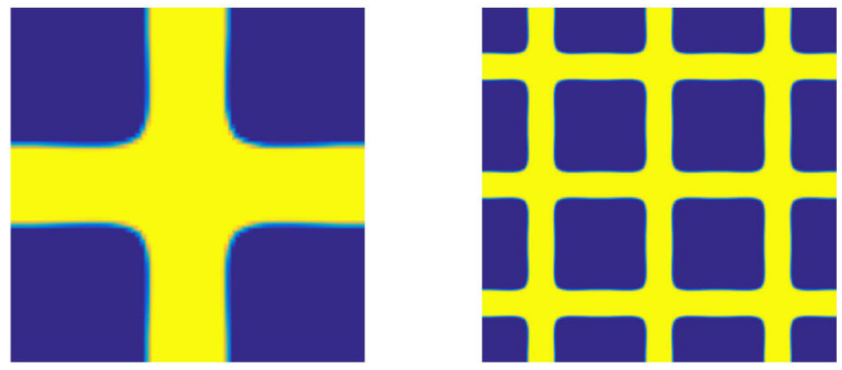

b $\delta_{4}=-0.1$
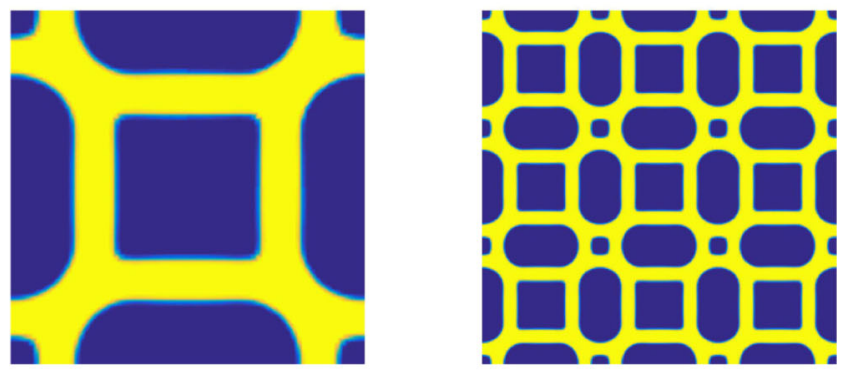

c $\delta_{r n d}=0.1$
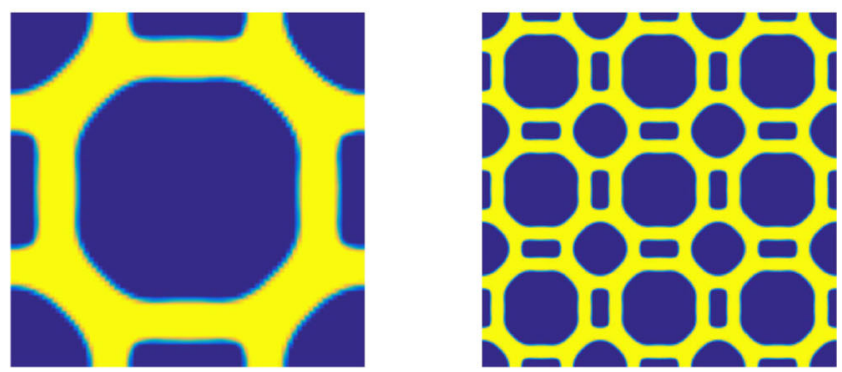

Fig. 6 Undeformed optimized unit cells (left) and array of $3 \times 3$ unit cells (right)

$123 \%$ more specific viscoplastic energy than a fully solid unit cell in which $\hat{c}=1$.

Next, the optimization is performed using a random initial 8-fold symmetric perturbation $\delta_{r n d}=0.1$. The optimized design, cf. Fig. 6c, consists of an octagonal frame with connected bars aligned in the loading directions. The energy absorption of this design is also slightly (1.1\%) lower than that of Design $6 a$.

Designs $6 \mathrm{a}-\mathrm{c}$ are alternate solutions to the optimization problem. And as shown in Fig. 7, the resulting energy absorption capabilities are not equal. In the upper part of this figure, we also see that the convergence towards a distinct solid-void design is slower for Designs $6 \mathrm{~b}-\mathrm{c}$ as compared with Design 6a. It is also seen that only minor design changes are observed when the thresholding parameter $\beta_{H}$ is increased after optimization iteration 80 , but the viscoplastic work increases as the intermediate values of $\hat{c}$ are thresholded closer towards 0 and 1 .

We conclude that that the initial design choice is crucial, and that several local maxima to this optimization problem exist. 

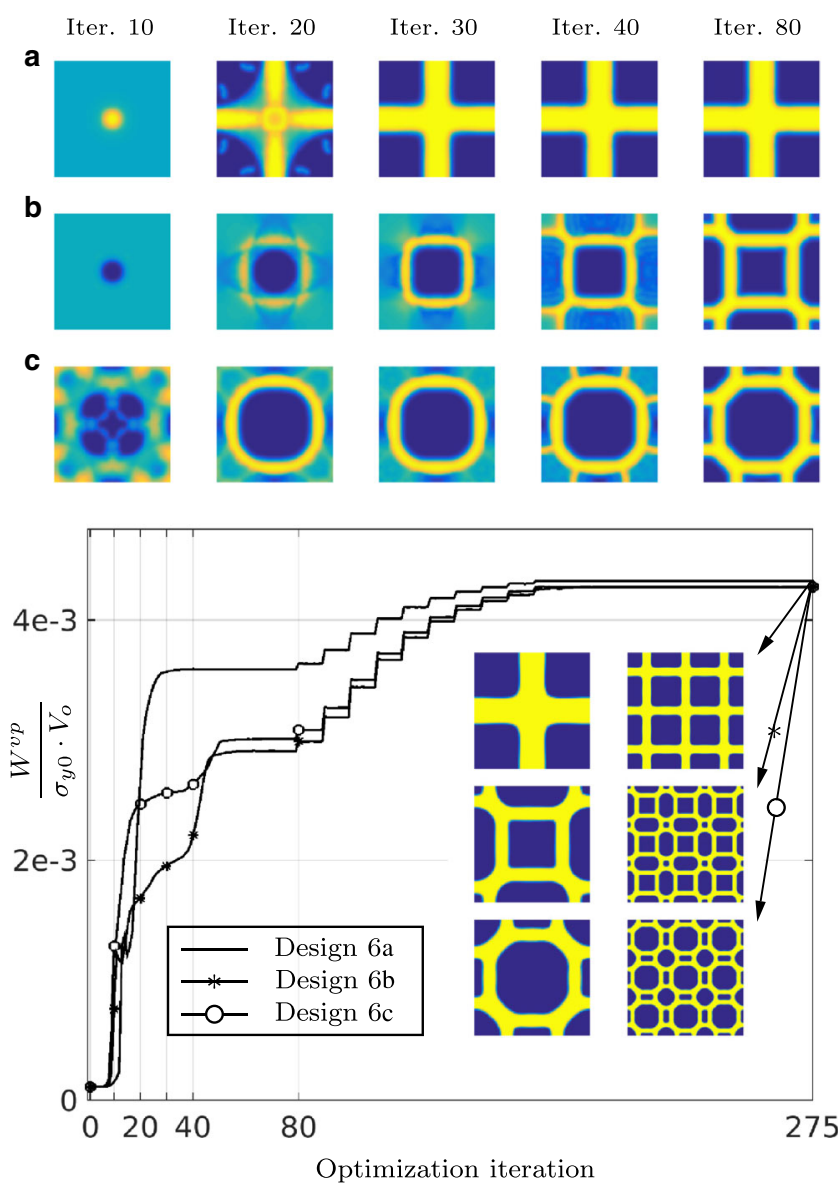

Fig. 7 Topology evolutions of Designs 6a-6c (top) and normalized viscoplastic work $W^{v p} /\left(\sigma_{y 0} V_{o}\right)$ versus optimization iteration, corresponding to the solid, solid-star, and solid-circle lines, respectively (bottom)

\subsection{Varying biaxial load paths}

Next, we demonstrate the importance of the path-dependent material model for material design under biaxial load cases. We consider the same final load state $\bar{u}_{x x}=\bar{u}_{y y}=0.01 \mathrm{~mm}$ at $t=T^{f}$ as for the previous optimization case, but vary the load path. Specifically, we use the same initial design $\delta_{4}=$ 0.1 and compare the optimized Design 6a, obtained with the loading conditions $\bar{u}_{x x}(t)=\bar{u}_{y y}(t)=t / T^{f} \cdot 0.01 \mathrm{~mm}$, to several other loading scenarios with $\bar{u}_{x x}(t) \neq \bar{u}_{y y}(t)$ for $0<t<T^{f}$. Figure 8 shows the resulting optimized designs with corresponding prescribed trajectories $\bar{u}_{x x}(t)$ and $\bar{u}_{y y}(t)$ which are split into two piecewise linear trajectories

$f_{\bar{u}}(t, a)= \begin{cases}a \cdot t / T^{f}, & t / T^{f}<0.5, \\ a-0.01+(0.02-a) \cdot t / T^{f}, & t / T^{f} \geq 0.5,\end{cases}$

where the parameter $a$ values corresponding to each design appear in Table 1. a Load path 1

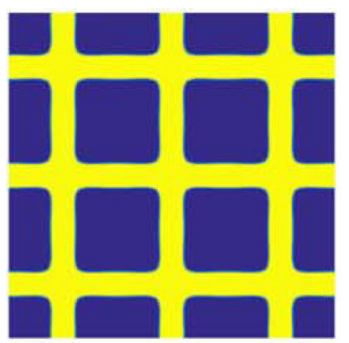

b Load path 2

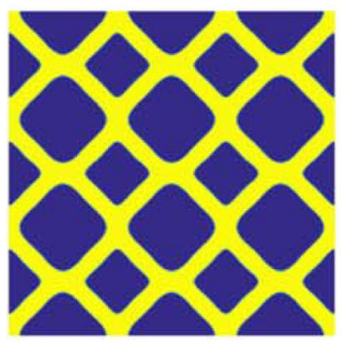

c Load path 3

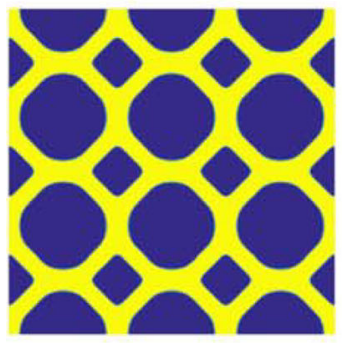

d Load path 4

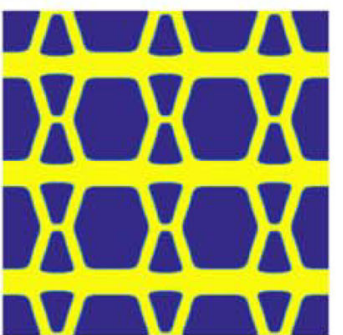

e Load path 5
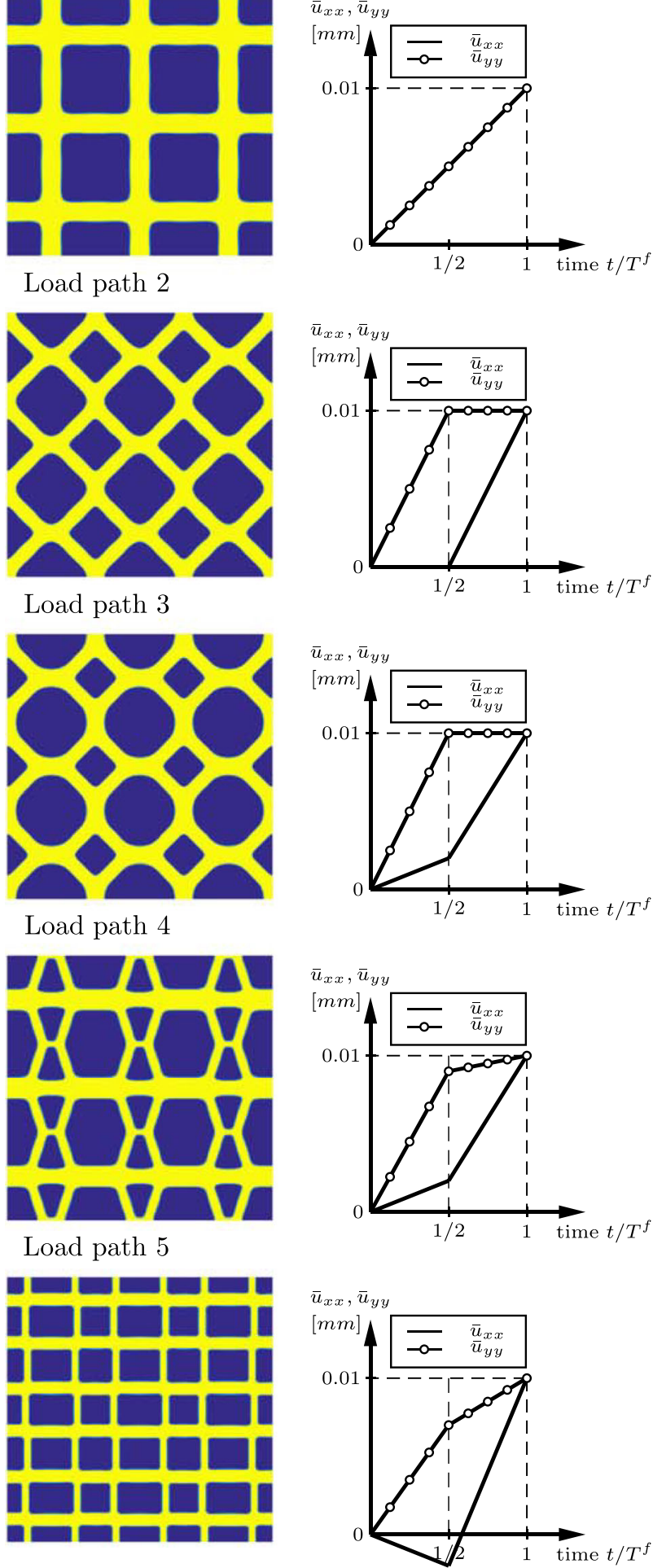

Fig. 8 Undeformed optimized array of $3 \times 3$ unit cells (left) with corresponding load path (right)

As seen in Fig. 8, different loading trajectories generate different optimized microstructures. This study clearly demonstrates the importance of including the 
Table 1 Parameter $a$ values for nonproportional biaxial loads

\begin{tabular}{lll}
\hline Design & $\bar{u}_{x x}$ & $\bar{u}_{y y}$ \\
\hline $8 \mathrm{~b}$ & 0 & 0.020 \\
$8 \mathrm{c}$ & 0.004 & 0.020 \\
$8 \mathrm{~d}$ & 0.004 & 0.018 \\
$8 \mathrm{e}$ & -0.006 & 0.014 \\
\hline
\end{tabular}

path-dependent model for maximation of viscoplastic energy in microstructural design.

\subsection{Displacement constraints}

In the following examples, we design microstructures with nearly zero transverse contraction when subjected to uniaxial tensile loading, e.g., for the $\bar{u}_{x x}$ loading, the constraint

a $\quad \dot{\varepsilon}_{x x}=10^{-15} s^{-1}$

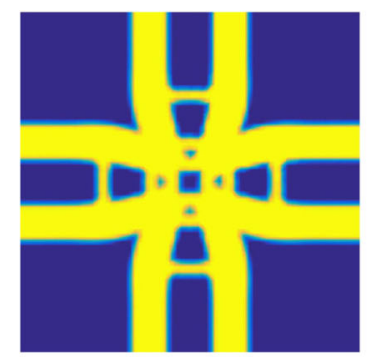

b $\dot{\varepsilon}_{x x}=100 s^{-1}$

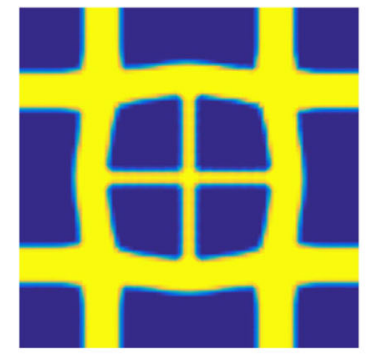

c $\quad \dot{\varepsilon}_{x x}=500 s^{-1}$
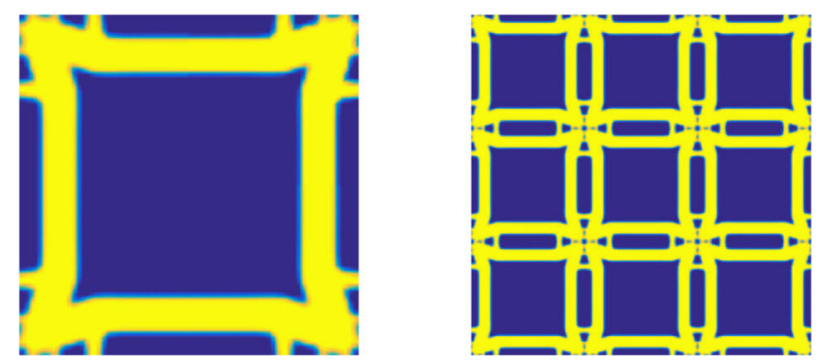

Fig. 9 Undeformed optimized designs (left) and array of $3 \times 3$ unit cells (right) under the load $\bar{u}_{x x}=0.01 \mathrm{~mm}$

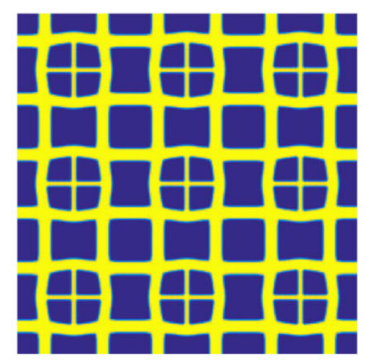

$g_{\bar{u}_{y y}}$ is included in the optimizations with the target trajectory $\bar{u}_{y y}^{*}=0 \forall t_{n} \in\left[0, T^{f}\right]$. To avoid constraining both the transverse and $z$-component displacements, plane stress conditions are used, i.e., periodicity is not enforced on the $z$-faces. An initial design with $\delta_{r n d}=0.002$ is used. For the cases where viscous effects are studied, i.e., when $\dot{\varepsilon} \neq 10^{-15} s^{-1}$, the loading rate is ramped up from $\dot{\varepsilon}=0.5 s^{-1}$ to the specified final value during the first two optimization iterations. This avoids the possibility that the initial design response is nearly elastic which we found otherwise hinders the optimization.

\subsubsection{Influence of strain rate and load magnitude}

In this example, we design material microstructures that perform equally under two uniaxial loads $\bar{u}_{x x}$ and $\bar{u}_{y y}$ by imposing the 8-fold domain symmetry illustrated in Fig. $2 \mathrm{~b}$. Thus, the computational effort is eased as only the single uniaxial load case $\bar{u}_{x x}$ needs be simulated. The effect of the load magnitude and loading rate on the microstructural topology is demonstrated by performing five different optimizations (Figs. 9 and 11). In the first three Designs 9a-9c are optimized under the moderate loads $\bar{u}_{x x}=0.01$ $m m$, with slow $\dot{\varepsilon}_{x x}=10^{-15} \mathrm{~s}^{-1}$, intermediate $\dot{\varepsilon}_{x x}=100$ $s^{-1}$, and fast $\dot{\varepsilon}_{x x}=500 s^{-1}$ load rates (cf. Section 7.1 and Fig. 3).

We compare the performance of these three designs by exposing them to the same load magnitude $\bar{u}_{x x}=0.01 \mathrm{~mm}$ and fast loading rate $\dot{\varepsilon}_{x x}=500 \mathrm{~s}^{-1}$, and measure their corresponding transverse contraction $-\bar{u}_{y y}$, cf. Fig. 10.

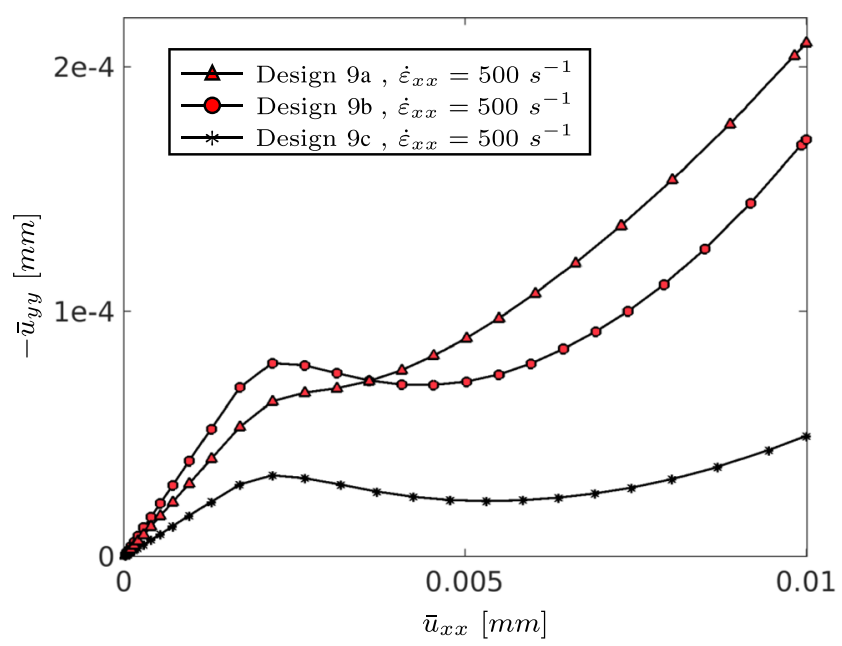

Fig. 10 Contraction $-\bar{u}_{y y}$ versus load $\bar{u}_{x x}$ for Designs $9 \mathrm{a}-\mathrm{c}$ under the same $\bar{u}_{x x}=0.01 \mathrm{~mm}$ load case with $\dot{\varepsilon}=500 \mathrm{~s}^{-1}$. Top two curves: responses of Designs $9 \mathrm{a}$ and $9 \mathrm{~b}$, which are not optimized for the fast $\dot{\varepsilon}=500 \mathrm{~s}^{-1}$ load rate. Both Design 9a and Design 9b violate $g_{\bar{u}_{y y}} \leq 0$. Bottom curve: response of Design 9c which satisfies $g_{\bar{u}_{y y}} \leq 0$ 
In this figure we see that, although Designs 9a and 9c have a similar microstructure, Design 9a violates the contraction constraint (35), cf. the solid-triangle line in Fig. 10. Design 9b, corresponding to the solid-circle line of Fig. 10, also contracts more than the allowed tolerance $\delta_{\bar{u}}=3 \cdot 10^{-3} \bar{u}_{x x}=3 \cdot 10^{-5} \mathrm{~mm}$. Figure 10 also shows that the contraction of all three designs is linearly increasing for loads $\bar{u}_{x x}$ less than approximately $0.002 \mathrm{~mm}$ but is nonlinear for higher loads $\bar{u}_{x x}>0.002 \mathrm{~mm}$. Clearly, the linear/nonlinear contraction versus load is a consequence of elastic and viscoplastic material response.

The last two optimizations are performed for a higher load $\bar{u}_{x x}=0.05 \mathrm{~mm}$, at slow $\dot{\varepsilon}_{x x}=10^{-15} \mathrm{~s}^{-1}$ and fast $\dot{\varepsilon}_{x x}=500$ $s^{-1}$ loading rates. The optimized designs are shown in Fig. 11. Although no obvious similarities can be seen from the optimized unit cells (cf. the left column of Fig. 11), these two optimizations generate two microstructures with similar topology as seen in the right column of Fig. 11. Both Designs $11 \mathrm{a}$ and $11 \mathrm{~b}$ satisfy the displacement constraint as shown by the center solid-circle and solid-triangle lines of Fig. 12a.

We further analyze the performance of the two Fig. 11 designs by plotting their corresponding macroscopic forcedisplacement curves, cf. Fig. 12b. The two center solidcircle and solid-triangle lines correspond to the macroscopic force-displacement responses of Designs 11a and 11b, respectively. As expected, Design 11b which is optimized

a $\dot{\varepsilon}_{x x}=10^{-15} s^{-1}$
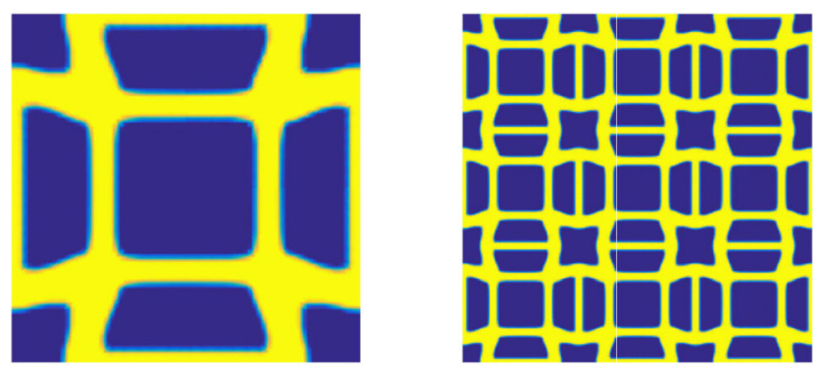

b $\quad \dot{\varepsilon}_{x x}=500 s^{-1}$
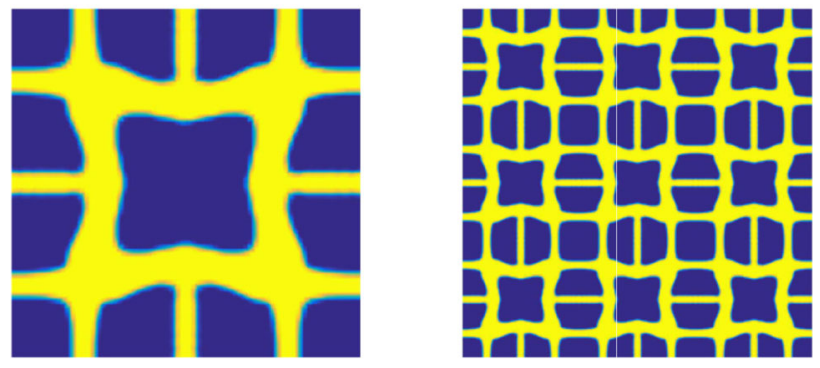

Fig. 11 Undeformed optimized designs (left) and array of $3 \times 3$ unit cells (right) under the load $\bar{u}_{x x}=0.05 \mathrm{~mm}$ for a higher loading rate is exposed to higher macroscopic forces.

Next, we simulate the responses of Designs $11 \mathrm{a}$ and $11 \mathrm{~b}$ at the load $\bar{u}_{x x}=0.05 \mathrm{~mm}$, but with interchanged loading rates, i.e., Designs $11 \mathrm{a}$ and $11 \mathrm{~b}$ are subjected to the load rates $\dot{\varepsilon}=500 s^{-1}$ and $\dot{\varepsilon}=10^{-15} s^{-1}$, respectively. Results of these simulations are displayed with red lines in Fig. $12 \mathrm{a}$ and $\mathrm{b}$.

The bottom two curves of Fig. 12b present the forcedisplacement responses of Designs $11 \mathrm{a}$ and $11 \mathrm{~b}$ when subjected to the slow loading rate. As expected, Design $11 \mathrm{~b}$ which is not optimized for this load rate performs worse than Design 11a; it supports less force and violates the displacement constraint, in fact it has negative transverse contraction. Surprisingly, Design 11a supports more force
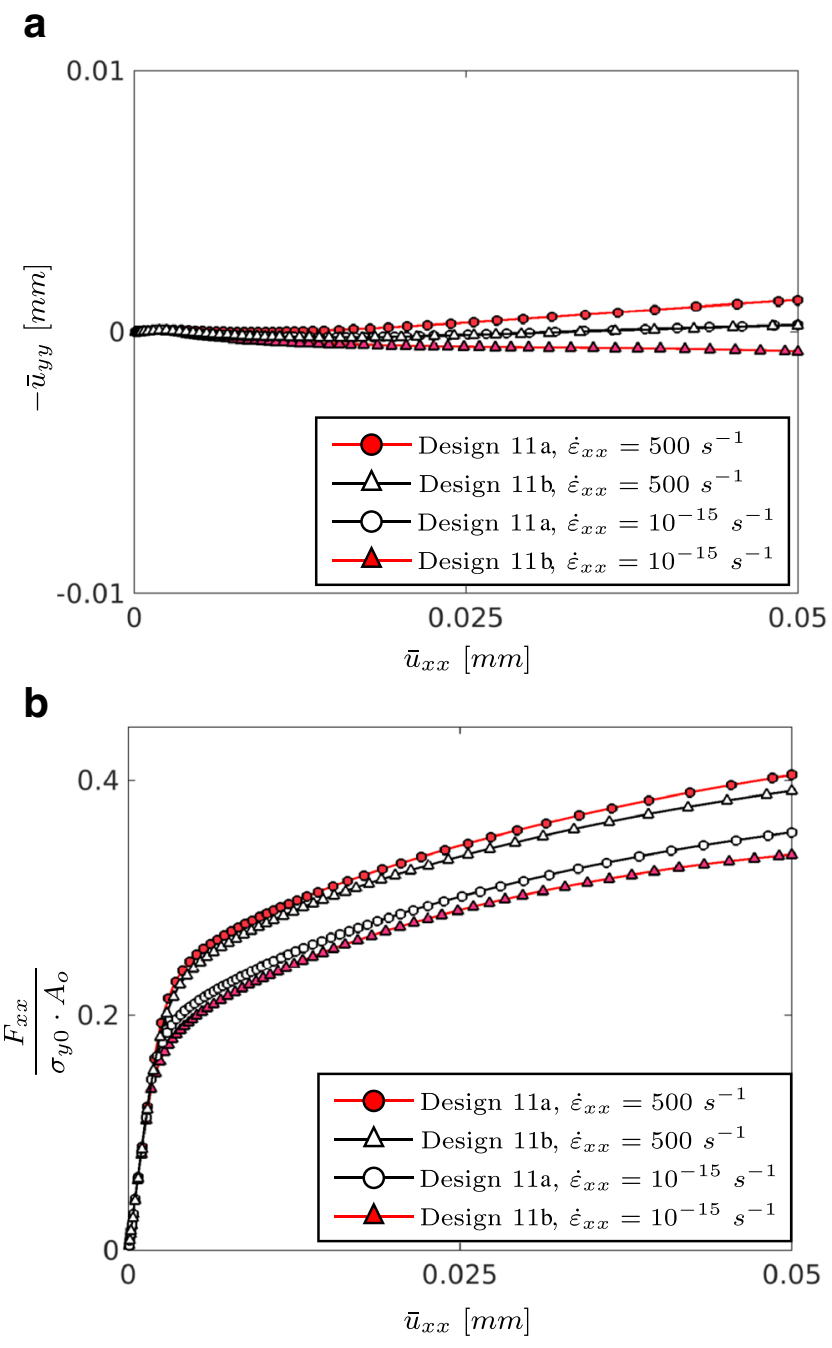

Fig. 12 Performance of Designs $11 \mathrm{a}$ and $11 \mathrm{~b}$. a contraction $-\bar{u}_{y y}$ versus load $\bar{u}_{x x}$ and $\mathbf{b}$ normalized macroscopic force $F_{x x} /\left(\sigma_{y 0} A_{o}\right)$ versus load $\bar{u}_{x x}$. The red lines with filled markers correspond to responses obtained from simulations with interchanged loading rates 
and hardens more than Design 11b when it is exposed to a fast loading rate. However, it violates the displacement constraint. These simulations show that more contraction leads to higher viscoplastic energy absorption. They also demonstrate that it is necessary to include rate effects in the optimization of viscoplastic microstructures.

We also compare the performance of Designs 9a and 11a by exposing them to the same load $\bar{u}_{x x}=0.05 \mathrm{~mm}$ and slow $\dot{\varepsilon}_{x x}=10^{-15} s^{-1}$ load rate. Figure 13 a shows that Design 9a which is optimized for the moderate load level $\bar{u}_{x x}=0.01 \mathrm{~mm}$ performs slightly better than Design 11a which is optimized for the $\bar{u}_{x x}=0.05 \mathrm{~mm}$ load, i.e., in this example, the energy absorption capability is not improved when optimizing for high load magnitudes.

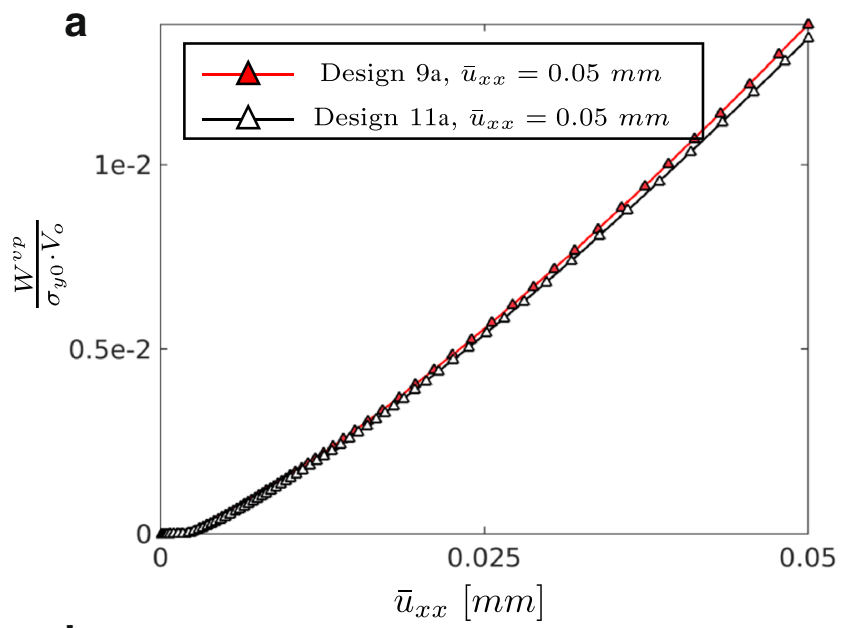

b

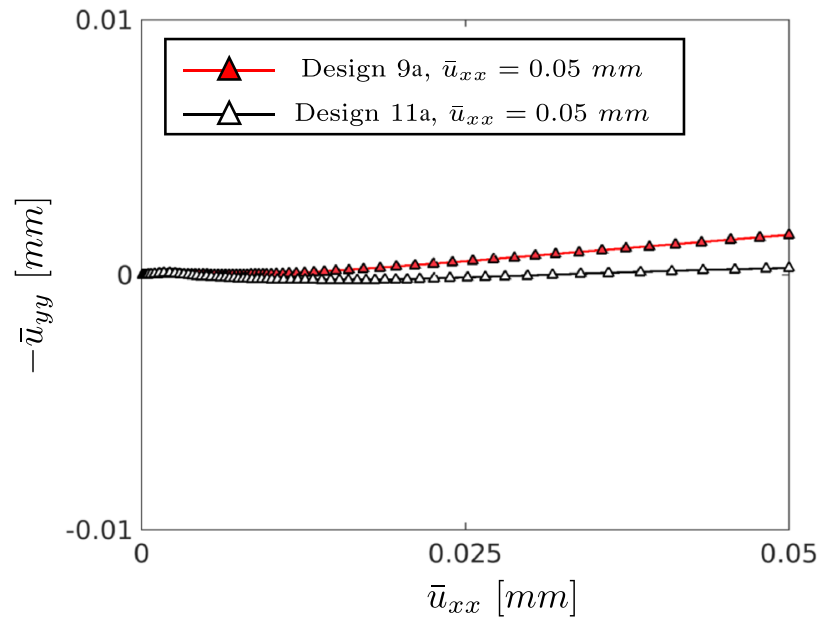

Fig. 13 a Normalized viscoplastic energy absorption versus load $\bar{u}_{x x}$ and $\mathbf{b}-\bar{u}_{y y}$ versus $\bar{u}_{x x}$, for Designs 11a and 9a. Design 9a, optimized for $\bar{u}_{x x}=0.01 \mathrm{~mm}$, absorbs $2.7 \%$ more viscoplastic energy but also has 6.5 times higher RMSD of $\bar{u}_{y y}$ from the target $\bar{u}_{y y}^{*}=0$ during the $\bar{u}_{x x}=0.05 \mathrm{~mm}$ load than Design 11a (which is optimized for $\left.\bar{u}_{x x}=0.05 \mathrm{~mm}\right)$
However, Design 11a fails to satisfy the displacement constraint when subjected to the larger load, cf. Fig. 13b, which shows strain-dependent contraction in the $\bar{u}_{x x} \in$ [0.01, 0.05] $\mathrm{mm}$ interval, in contrast to Design 11a that exhibits nearly zero contraction throughout the load interval. Similar to the results presented in Fig. 12, this comparison shows that higher contraction leads to increased viscoplastic energy absorption.

\subsubsection{Multiple uniaxial tensile loads: influence of load magnitude}

In this final example, we optimize the unit cell under multiple load cases. Specifically, both longitudinal and transversal load cases are simulated using 4-fold domain symmetry with symmetry boundary conditions (cf. Fig. 2a). We include both the $g_{\bar{u}_{y y}}$ and $g_{\bar{u}_{x x}}$ constraints in the optimization.

The objective is to maximize the geometric mean of the viscoplastic energy absorption, $\left(W_{x}^{v p} \cdot W_{y}^{v p}\right)^{1 / 2}$, where $W_{x}^{v p}$ and $W_{y}^{v p}$ are the energies absorbed under the uniaxial loads

a

$\bar{u}_{x x}=0.01 \mathrm{~mm}$
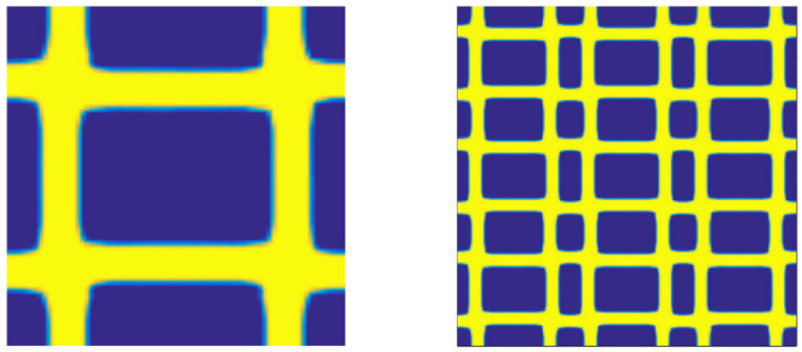

b $\bar{u}_{x x}=0.05 \mathrm{~mm}$
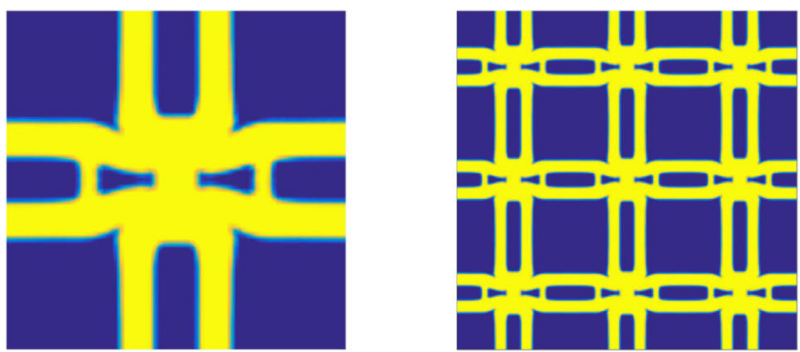

c $\bar{u}_{x x}=0.10 \mathrm{~mm}$
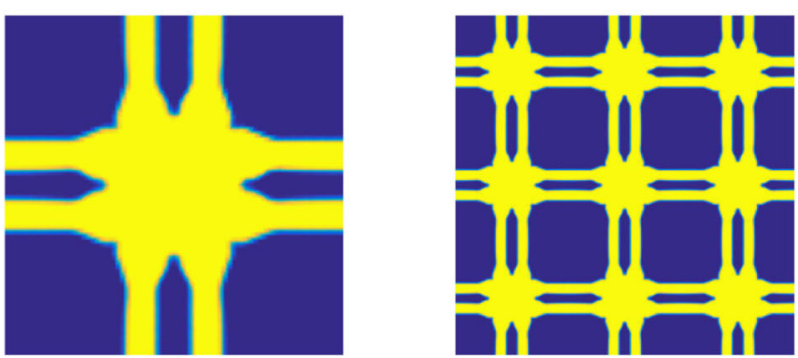

Fig. 14 Undeformed optimized designs (left) and array of $3 \times 3$ unit cells (right) 


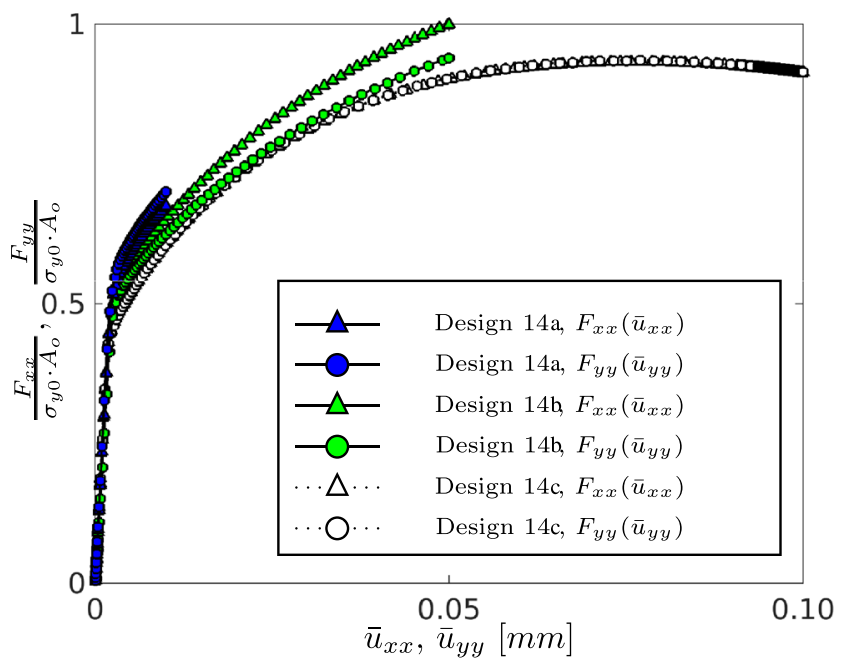

Fig. 15 Normalized macroscopic forces $F_{x x} /\left(\sigma_{y 0} A_{o}\right)$ and $F_{y y} /\left(\sigma_{y 0} A_{o}\right)$ versus load $\bar{u}_{x x}$ and $\bar{u}_{y y}$ for Designs $14 \mathrm{a}-\mathrm{c}$

$\bar{u}_{x x}$ and $\bar{u}_{y y}$, respectively. The optimization is performed for the load magnitudes $0.01 \mathrm{~mm}, 0.05 \mathrm{~mm}$, and $0.10 \mathrm{~mm}$ with the slow loading rate $\dot{\varepsilon}=10^{-15} s^{-1}$, all initiated with the same design. The optimized designs are illustrated in Fig. 14. The microstructural Design 14a, optimized for the moderate load magnitude $0.01 \mathrm{~mm}$, consists of a rectangular pattern of material with slight offsets to satisfy the near zero contraction constraints, whereas Designs $14 \mathrm{~b}$ and 14c, optimized for the higher load magnitudes $0.05 \mathrm{~mm}$ and 0.10 $\mathrm{mm}$, respectively, consist of more complex topologies. All three designs satisfy the displacement constraints.

The macroscopic force-displacement responses of Designs 14a-14c, plotted in Fig. 15, show that Design

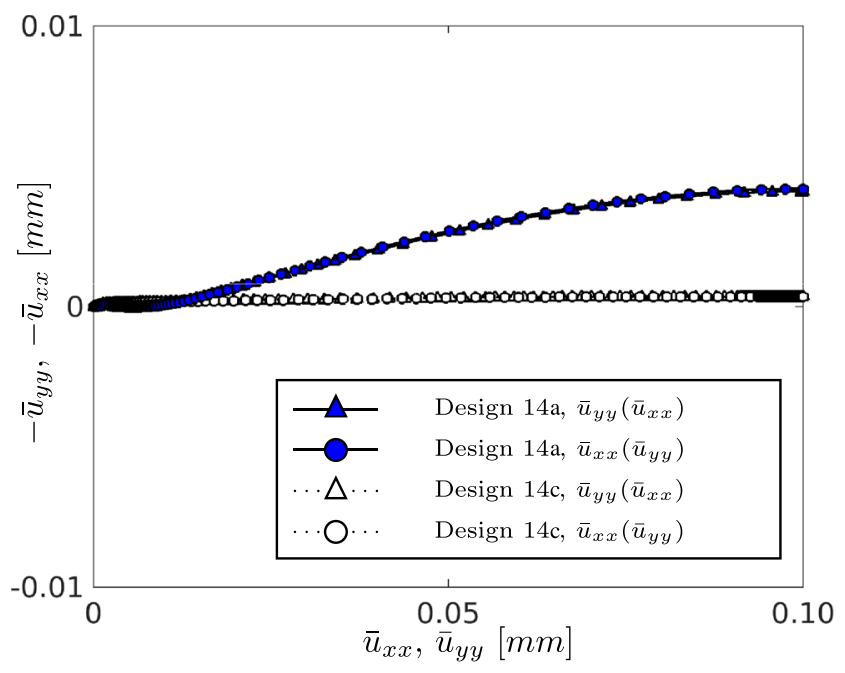

Fig. 16 Contraction $-\bar{u}_{y y}$ and $-\bar{u}_{x x}$ versus load $\bar{u}_{x x}$ and $\bar{u}_{y y}$ for Designs $14 \mathrm{a}$ and $14 \mathrm{c}$

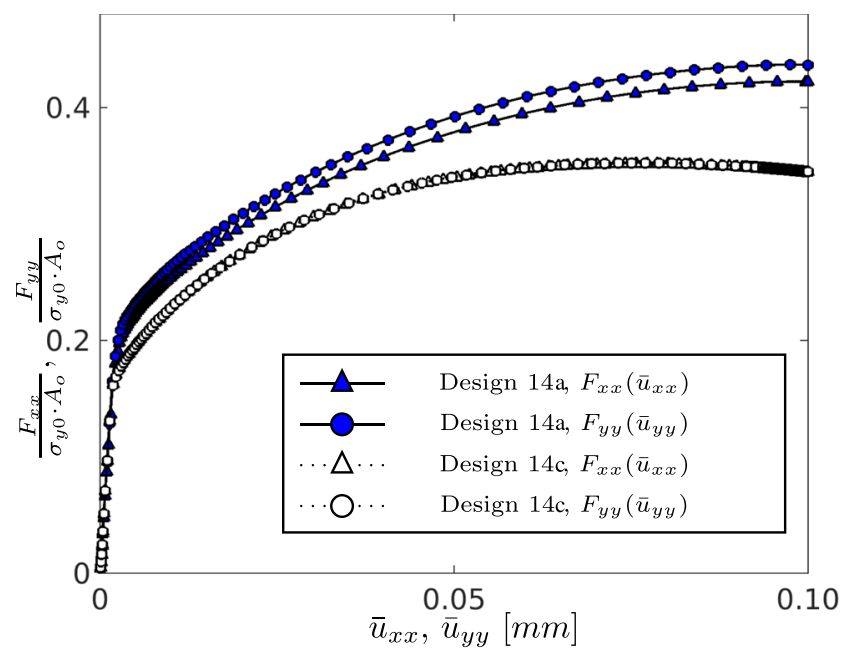

Fig. 17 Normalized macroscopic forces $F_{x x} /\left(\sigma_{y 0} A_{o}\right)$ and $F_{y y} /\left(\sigma_{y 0} A_{o}\right)$ versus loads $\bar{u}_{x x}$ and $\bar{u}_{y y}$ for Designs $14 \mathrm{a}$ and $14 \mathrm{c}$

14a supports higher forces and thus can dissipate more viscoplastic energy than Designs $14 \mathrm{~b}$ and $14 \mathrm{c}$ in the load interval $[0,0.01] \mathrm{mm}$, for both the $\bar{u}_{x x}$ and $\bar{u}_{y y}$ loads. It is also seen that Design 14b supports higher forces than Design $14 \mathrm{c}$ in the load interval [0, 0.05] $\mathrm{mm}$ for both loads.

Finally, we compare Designs 14a and 14c, optimized for the load magnitudes $0.01 \mathrm{~mm}$ and $0.10 \mathrm{~mm}$, respectively, by exposing them to the same high load interval $[0,0.10] \mathrm{mm}$. Figure 16 shows pronounced difference in the contraction versus load, e.g., $-\bar{u}_{y y}$ versus $\bar{u}_{x x}$ between Designs $14 \mathrm{a}$ and $14 \mathrm{c}$. Indeed, Design 14a has nearly zero contraction in the $[0,0.01] \mathrm{mm}$ load interval for which it was designed but shows a considerable strain-dependent contraction in the $(0.01,0.10] \mathrm{mm}$ load interval.

Figure 17 shows the normalized macroscopic forcedisplacement responses for Designs 14a and 14c. Here it is seen that Design 14c, optimized for the high load magnitude $0.10 \mathrm{~mm}$, softens for loads $\bar{u}_{x x}, \bar{u}_{y y}>0.08 \mathrm{~mm}$, whereas Designs 14a exhibits macroscopic hardening. Similar to the previous studies, this example demonstrates that higher contraction (cf. Fig. 16) leads to more supportive structures (cf. Fig. 17) and thus higher viscoplastic energy absorption.

\section{Conclusions}

We have established a topology optimization framework for designing periodic microstructural composite materials for maximum viscoplastic energy absorption. Constraints have also been imposed on designs such that, under uniaxial tensile loads, their macroscopic transverse contraction is close to zero. The optimization problems are solved using 
the MMA scheme and sensitivities required to drive the algorithm are obtained using the adjoint method. The effects of strain rate, load magnitude, and path-dependency are shown by optimizing designs under several complex macroscopic load cases. It is clear that these effects are of great importance on the optimized microstructural design. The present approach allows each material point in a macroscopic structure to be tailored to the loading rate, load path, and load magnitude it experiences.

The unit cell that characterizes the design domain has been discretized with 3D finite elements. This formulation enables a smooth transition to large-scale 3D problems by relaxing of the plane strain and plane stress assumptions. In future work, we will apply parallel programming to the framework to efficiently solve such large-scale problems.

Acknowledgments The authors would like to thank Prof. Krister Svanberg for providing the MMA code.

Funding information Open access funding provided by Lund University. This work was partially performed under the auspices of the US Department of Energy by Lawrence Livermore Laboratory under contract DE-AC52-07NA27344, cf. ref number LLNL-CONF-717640. The financial support from the Swedish research council (grant nbr. 2015-05134) and the Swedish Energy Agency (grant nbr. 48344-1) is gratefully acknowledged.

\section{Compliance with ethical standards}

Conflict of interest The authors declare that they have no conflict of interest.

Replication of results The results presented in this article are produced from our Fortran in-house code which uses the Fortran implementation of Prof. Krister Svanbergs MMA optimization solver. We use the Intel Fortran Composer XE 2011 compiler, direct sparse Intel MKL PARDISO solver and utilize multithreading with OpenMP. The geometry input file is generated in Abaqus 6.13. To encourage the replication of the results in this paper, a detailed pseudocode of the optimization framework is given in Algorithms 1 and 2. The data for the initial designs used to generate the results in Section 7.5 and in the Appendix are not included, as they are generated by the pseudorandom number generator and are dependent on the time seeds.

Open Access This article is licensed under a Creative Commons Attribution 4.0 International License, which permits use, sharing, adaptation, distribution and reproduction in any medium or format, as long as you give appropriate credit to the original author(s) and the source, provide a link to the Creative Commons licence, and indicate if changes were made. The images or other third party material in this article are included in the article's Creative Commons licence, unless indicated otherwise in a credit line to the material. If material is not included in the article's Creative Commons licence and your intended use is not permitted by statutory regulation or exceeds the permitted use, you will need to obtain permission directly from the copyright holder. To view a copy of this licence, visit http:// creativecommonshorg/licenses/by/4.0/.

\section{Appendix: Sensitivity verification}

To verify correct implementation of the adjoint sensitivity analysis presented in Section 6.2, we compare the sensitivities obtained by the adjoint method with numerical sensitivities obtained by the central difference method (CDM). We use 4-fold domain symmetry and the corresponding symmetry boundary conditions of Fig. 2a to discretize a quadrant of the unit cell by $5 \times 5 \times 1$ tri-linear brick elements, and subject it to biaxial loading conditions (cf. Fig. 5) with load path illustrated in Fig. 8e. To ensure large deformations and viscoplastic response, the final prescribed displacements $\bar{u}_{x x}$ and $\bar{u}_{y y}$ are increased to $0.05 \mathrm{~mm}$ and the load duration is decreased to $T^{f}=0.01 s$; this is done by scaling the Fig. 8 load path. Each design variable value is given by the random number generator through $\phi_{e}=\operatorname{rand}(0,1)$, and for the PDE filtering, we set $l_{o}=0.25 \mathrm{~mm}$. The adaptive time-stepping is omitted for this analysis that instead uses 40 equally spaced load increments.

Figure 18 shows that the sensitivities obtained by the adjoint (solid, blue curve) and the central difference method (dashed, red curve) are almost equal; the relative error between the two methods is smaller than the perturbation size $\Delta h=10^{-6}$ as shown by the dotted line; hence, the derivation and implementation of the adjoint sensitivities are correct.

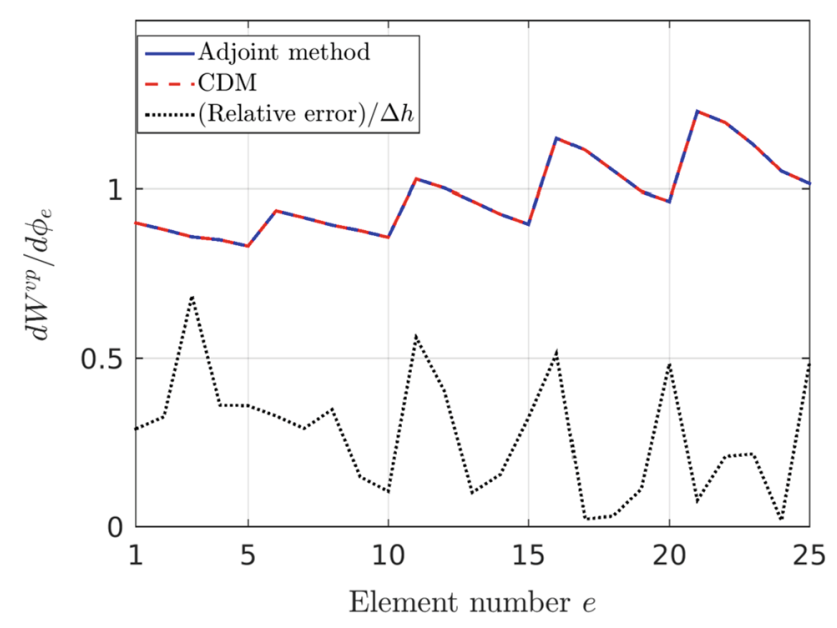

Fig. 18 Comparison of sensitivities obtained by the adjoint method and the central difference method (CDM) 


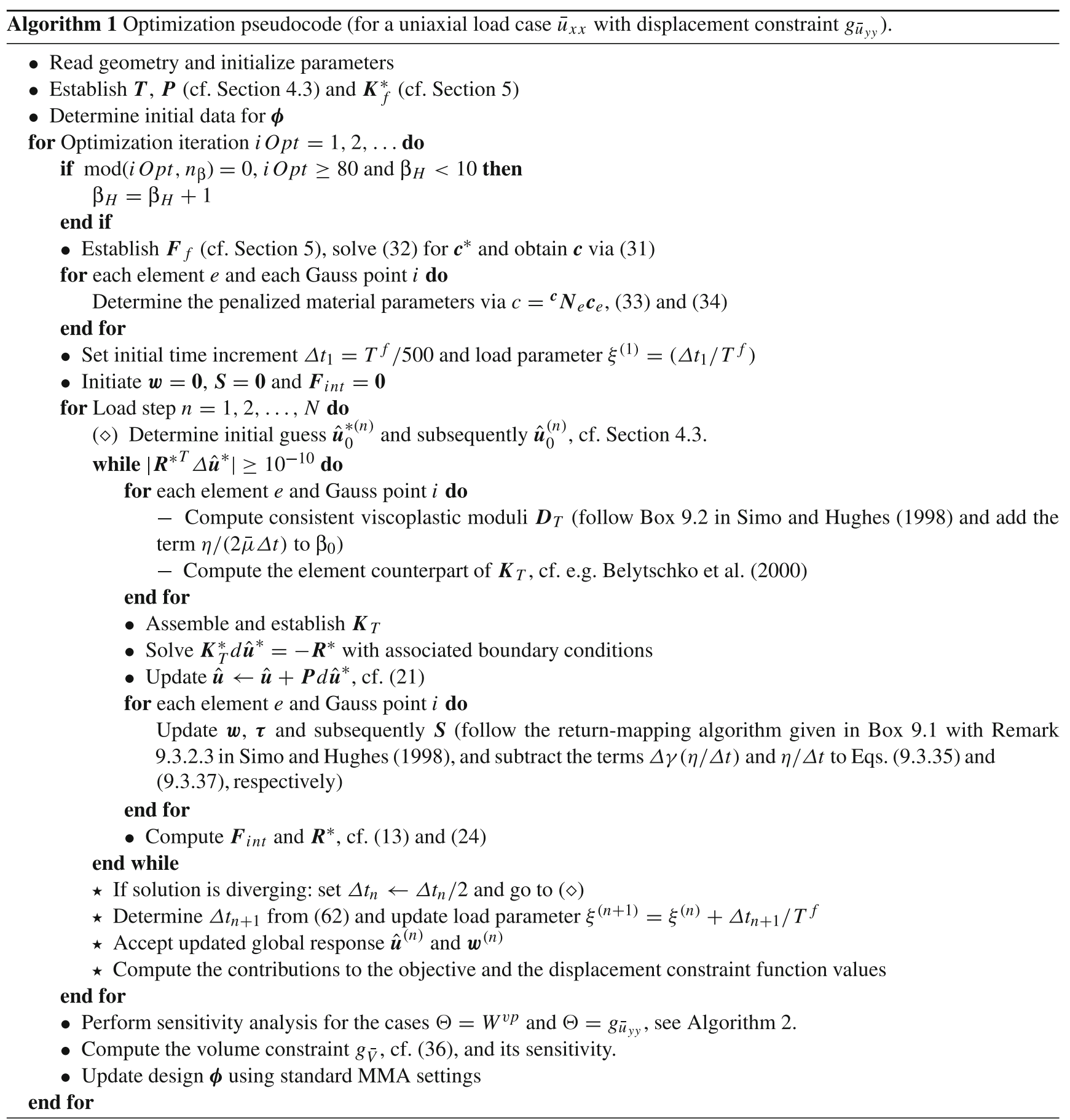




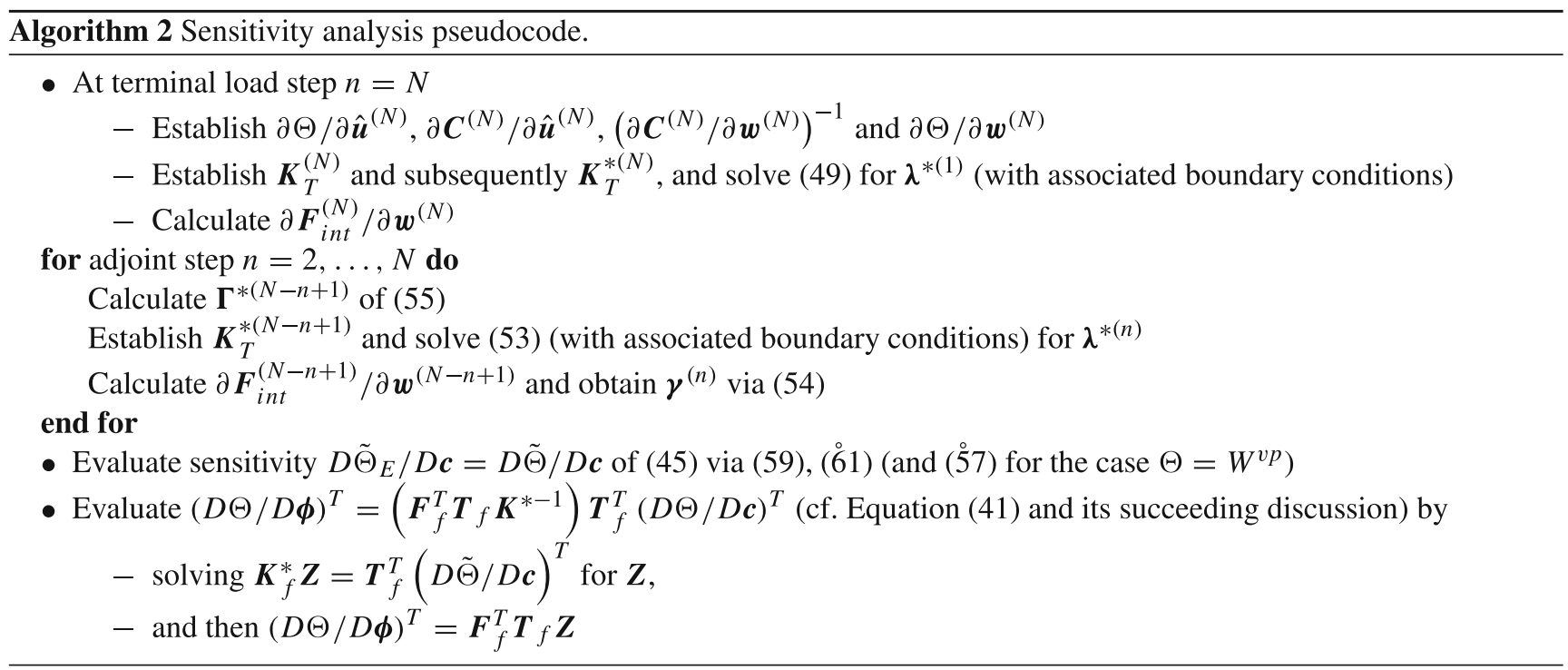

\section{References}

Alberdi R, Khandelwal K (2019) Design of periodic elastoplastic energy dissipating microstructures. Struct Multidiscipl Optim 59(2):461-483. https://doi.org/10.1007/s00158-018-2076-2

Amir O (2017) Stress-constrained continuum topology optimization: a new approach based on elasto-plasticity. Struct Multidiscipl Optim 55(5):1797-1818. https://doi.org/10.1007/s00158-016-1618-8

Andreasen CS, Andreassen E, Jensen JS, Sigmund O (2014) On the realization of the bulk modulus bounds for two-phase viscoelastic composites. J Mech Phys Solids 63:228-241. https://doi.org/10. 1016/j.jmps.2013.09.007

Belytschko T, Liu W, Moran B (2000) Nonlinear finite elements for continua and structures. Wiley

Bendsøe MP, Kikuchi N. (1988) Generating optimal topologies in structural design using a homogenization method. CMAME 71:197-224

Bendsoe MP, Sigmund O (2013) Topology optimization: theory, methods, and applications. Springer

Bogomolny M, Amir O (2012) Conceptual design of reinforced concrete structures using topology optimization with elastoplastic material modeling. Int J Numer Meth Engng 90(13):1578-1597

Borgqvist E, Wallin M (2013) Numerical integration of elastoplasticity coupled to damage using a diagonal implicit runge-Kutta integration scheme. Int J Damage Mech 22:68-94

Chen W, Xia L, Yang J, Huang X (2018) Optimal microstructures of elastoplastic cellular materials under various macroscopic strains. Mech Mater 118:120-132. https://doi.org/10.1016/j.mechmat. 2017.10.002

Deaton JD, Grandhi RV (2014) A survey of structural and multidisciplinary continuum topology optimization: post 2000 . Struct Multidiscip Optim 49(1):1-38

Fritzen F, Xia L, Leuschner M, Breitkopf P (2016) Topology optimization of multiscale elastoviscoplastic structures. Int J Numer Methods Eng 106(6):430-453. https://doi.org/10.1002/nme.5122

Guest JK, Prévost JH, Belytschko T (2004) Achieving minimum length scale in topology optimization using nodal design variables and projection functions. Int J Numer Meth Engng 61(2):238-254
Huang X, Radman A, Xie Y (2011) Topological design of microstructures of cellular materials for maximum bulk or shear modulus. Comput Mater Sci 50(6):1861-1870. https://doi.org/10.1016/j. commatsci.2011.01.030

Ivarsson N, Wallin M, Tortorelli D (2018) Topology optimization of finite strain viscoplastic systems under transient loads. Int $\mathbf{J}$ Numer Methods Eng 114(13):1351-1367. https://doi.org/10.1002/ nme.5789

Kato J, Hoshiba H, Takase S, Terada K, Kyoya T (2015) Analytical sensitivity in topology optimization for elastoplastic composites. Struct Multidiscip Optim 52:507-526

Lazarov BS, Sigmund O (2011) Filters in topology optimization based on helmholtz-type differential equations. Int J Numer Meth Engng 86(6):765-781. https://doi.org/10.1002/nme.3072

Maute K, Schwarz S, Ramm E (1998) Adaptive topology optimization of elastoplastic structures. Struct Optim 15(2):81-91

Michaleris P, Tortorelli D, Vidal CA (1994) Tangent operators and design sensitivity formulations for transient non-linear coupled problems with applications to elastoplasticity. Int J Numer Meth Engng 37(14):2471-2499

Nakshatrala P, Tortorelli D (2015) Topology optimization for effective energy propagation in rate-independent elastoplastic material systems. Comput Methods Appl Mech Eng 295:305-326. https://doi.org/10.1016/j.cma.2015.05.004

Nakshatrala P, Tortorelli D, Nakshatrala K (2013) Nonlinear structural design using multiscale topology optimization. part i: Static formulation. Comput Methods Appl Mech Eng 261(262):167176. https://doi.org/10.1016/j.cma.2012.12.018

Pedersen CB (2004) Crashworthiness design of transient frame structures using topology optimization. Comput Methods Appl Mech Eng 193(6):653-678. https://doi.org/10.1016/j.cma.2003.11.001

Ristinmaa M, Ottosen NS (2000) Consequences of dynamic yield surface in viscoplasticity. Int J Solids Struct 37(33):4601-4622. https://doi.org/10.1016/S0020-7683(99)00158-4

Sigmund O (1994) Materials with prescribed constitutive parameters: an inverse homogenization problem. SS 31(17):2313-2329

Sigmund O (1995) Tailoring materials with prescribed elastic properties . Mech Mater 20(4):351-368. https://doi.org/10.1016/01676636(94)00069-7 
Sigmund O, Torquato S (1997) Design of materials with extreme thermal expansion using a three-phase topology optimization method. J Mech Phys Solids 45(6):1037-1067. https://doi.org/10.1016/ S0022-5096(96)00114-7

Simo JC, Hughes TJR (1998) Computational inelasticity, vol 7. Springer

Simo JC, Miehe C (1992) Associative coupled thermoplasticity at finite strains: formulation, numerical analysis and implementation. Comput Methods Appl Mech Eng 98:41-104

Simo JC, Taylor RL (1985) Consistent tangent operators for rateindependent elasto-plasticity. CMAME 48:101-118

Stolpe M, Svanberg K (2001) An alternative interpolation scheme for minimum compliance topology optimization. Struct Multidiscip Optim 22:116-124

Svanberg K (1987) The method of moving asymptotes - a new method for structural optimization. Int J Numer Meth Engng 24:359-373

Svanberg K (2002) A class of globally convergent optimization methods based on conservative convex separable approximations. SIAM J Optim 12(2):555-573

Träff E, Sigmund O, Groen JP (2019) Simple single-scale microstructures based on optimal rank-3 laminates. Struct Multidiscipl Optim 59(4):1021-1031. https://doi.org/10.1007/s00158-018-2180-3

Wallin M, Tortorelli D (2020) Nonlinear homogenization for topology optimization. Mechanics of Materials, pp 103324. https://doi.org/ 10.1016/j.mechmat.2020.103324

Wallin M, Jönsson V, Wingren E (2016) Topology optimization based on finite strain plasticity. Struct Multidiscip Optim 54(4):783-793
Wallin M, Ivarsson N, Tortorelli D (2018) Stiffness optimization of non-linear elastic structures. Comput Methods Appl Mech Eng 330:292-307. https://doi.org/10.1016/j.cma.2017.11.004

Wang F, Lazarov BS, Sigmund O, Jensen JS (2014) Interpolation scheme for fictitious domain techniques and topology optimization of finite strain elastic problems. CMAME 276:453-472

Wang F, Sigmund O, Jensen J (2014) Design of materials with prescribed nonlinear properties. J Mech Phys Solids 69:156-174. https://doi.org/10.1016/j.jmps.2014.05.003

Yvonnet J, Gonzalez D, He QC (2009) Numerically explicit potentials for the homogenization of nonlinear elastic heterogeneous materials. Comput Methods Appl Mech Eng 198(33):2723-2737. https://doi.org/10.1016/j.cma.2009.03.017

Zhang G, Khandelwal K (2019) Design of dissipative multimaterial viscoelastic-hyperelastic systems at finite strains via topology optimization. Int J Numer Methods Eng 119(11):1037-1068. https://doi.org/10.1002/nme.6083

Zhang G, Li L, Khandelwal K (2017) Topology optimization of structures with anisotropic plastic materials using enhanced assumed strain elements. Struct Multidiscipl Optim 55(6):19651988. https://doi.org/10.1007/s00158-016-1612-1

Publisher's note Springer Nature remains neutral with regard to jurisdictional claims in published maps and institutional affiliations. 